\title{
Die Bedeutung gewisser Nucleoproteïde für die oxydative Leistung der Zelle.
}

\author{
Von \\ Dr. W. Spitzer, \\ Breslau-Karlsbad.
}

In einer frïheren Arbeit ${ }^{1}$ ) babe ich den Versuch gemacht, gewisse an "todten" Geweben beobachtete und durch sie bedingte Oxydationsvorgänge unter einem gemeinsamen Gesichtspunkte zu betrachten und für dieselben eine theoretische Erklärung zu geben. Ich glaube nachgewiesen zu haben, dass wir auf Grund der vorliegenden Thatsachen eine Reihe ron Oxydationsprocessen, die in todten Substraten beobachtet werden können, wie die Glycolyse, die Zerlegung von $\mathrm{H}_{2} \mathrm{O}_{2}$, die synthetische Bildung von gewissen organischen Farbstoffen als analoge und im wesentlichen gleichartige Processe ansehen und auf die Wirkung gleicher Ursachen zuriekfithren können.

Wir haben in Anlehnung an die Theorie von M. T ra u be angenommen, dass jenen oxydativen Vorgängen eine eigenthümliche Fähigkeit der "todten“ Gewebe zu Grunde liegt, nämlich die, in Anwesenheit gewisser sonst schwer oxydabler Verbindungen den molecularen Sauerstoff der Luft zu „erregen“, zu ,activiren“, sein Moleciil in active Atome $z \mathfrak{u}$ spalten und mit Hülfe dieser jene „dysoxydablen" Verbindungen der Verbrennung zuzuführen. Wir stellen uns den Vorgang dieser „Erregung" oder „Uebertragang" genau so vor, wie wir ihn von den sogenannten katalytischen Oxydationen durch anorganische Sauerstoffüberträger,

1) Die zuckerzerstörende Kraft des Blutes und der Gewebe. Ein Beitrag zur Lehre von der Oxydationswirkung thierischer Gewebe. Pflüger's Archiv Bd. 60. 303. 1895. u. Berl. Klin. Wochenschrift 1894, 42. 
wie von der Verbrennung des itber Platinsehwamm geleiteten Wasserstoffs, der Zerlegung des $\mathrm{H}_{2} \mathrm{O}_{2}$ durch Platin, Palladium etc. kennen, wie er für ersteren Prozess durch die Gleichung:

$$
\begin{aligned}
& \mathrm{Pt}+\mathrm{H}_{2}+\mathrm{O}_{2}=\mathrm{PtO}+\mathrm{H}_{2} \mathrm{O} \\
& \mathrm{PtO}+\mathrm{H}_{2}=\mathrm{Pt}+\mathrm{H}_{2} \mathrm{O}
\end{aligned}
$$

seine schematische Erklärung findet.

Die Anschauung Tra $\mathrm{u}$ be's von der durch 0 Uebertragung wirkenden Oxydationskraft der Gewebe haben wir auch nach der Ricbtung hin erweitert, dass wir auch den zu oxydirenden Substraten bei jenem Vorgange eine wichtige active Rolle zutheilen, indem wir annehmen, dass der Ablauf des Processes nicht minder auch wesentlich abhängig erscheint von ihrer stärkeren oder schwächeren Fähigkeit, Sauerstoff an sich zu ziehen. Wir sehen somit die in ,todten" Substraten vor sich gehenden Oxydationsprocesse als - abgesehen von der unter allen Umständen nothwendigen Anwesenheit von Sauerstoff - abhängig von zwei Faktoren an, 1. der für das betreffende Organ stets constanten 0 iibertragenden Kraft, bez. der "Oxydationskraft" der in dem Organ entbaltenen Stoffe und 2. der nach den Bedingungen des Versuchs stets variablen zu oxydirenden Substanz beziehentlich deren Affinität zum Sauerstoff.

Wir haben des weiteren ausgeführt, wie auch wir in Uebereinstimmung mit allen anderen Antoren jene oxydativen Leistungen des Blutes und der Gewebe ausschliesslich an die sie bildenden Zellen gebunden fanden, denen das wirksame Princip durch einfache Extraction mit Wasser, wenn auch nicht völlig, so doch in grossem Maasse entzogen werden kann. Das weitere Studium der oxydirenden Wirkung der Gewebszellen brachte uns nunmehr eine Reihe recht interessanter nener Thatsachen.

I. Ueber die Intensität er oxydativen Leistung verschiedener Gewebe.

Es ersehien zunächst wïnschenswerth, die Energie der Oxydationskraft verschiedenartiger Gewebszellen and die Bedingungen ihrer Wirkung näher kennen zu lernen. Ich benitzte hierzu ihre Einwirkung auf Wasserstoffsuperoxyd und bediente mich hierbei folgender Versuchsanordnung. Unter ein graduirtes, mit Wasser gefülltes Eudiometer mündete ein gebogenes Glasrohr, das durch 
Die Bedeut. gewisser Nucleoproteide für d. oxydat. Leistung der Zelle. 617

einen Gummischlauch mit einem weithalsigen verschlossenen Kolben in Verbindung stand, der zur Aufnalme des Superoxyds und der zu prïfenden Snbstanz bestimmt war. Da die Zerlegung des $\mathrm{H}_{2} \mathrm{O}_{2}$ momentan eintritt, wurde, um einem Verluste des gebildeten und za messenden Sauerstoffs vorzubeugen, das zu prüfende Organgewebe in Lösung beziehentlich Aufschwemmung in den Kolben gebracht, und darauf ein kleines mit der $\mathrm{H}_{2} \mathrm{O}_{2}$-Lösung beschicktes Gläschen vorsichtig in denselben hinuntergelassen, der Kolben geschlossen and erst jetzt mit dem Eudiometer verbunden. Beim Umschutteln des Kolbens trat sofort die Zerlegung des $\mathrm{H}_{2} \mathrm{O}_{2}$ ein und der gebildete Sauerstoff beziehentlich die durch jenen verdrängte Luft trat in das Eudiometerrohr über und konnte dort von Minute zu Minute abgelesen werden. Von den jeweils geprüften Geweben wurden stets gleiche Mengen derselben, meistens $1 \mathrm{gr}$ in frischem Zustande verwendet, von der käuflichen ca 2-3\% igen Wasserstoffsuperoxydlösung wurden stets $10 \mathrm{ccm}$ zugesetzt. Dieselben wurden wegen ihres Gehaltes an $\mathrm{HCl}$ neutralisirt, wozu gewöhnlich 1 bis $1 \frac{1}{2}$ ccm $1 / 10 \mathrm{NaOH}$ nöthig waren. Zur Prüfung verwendet wurden fast alle Gewebe des Thierkörpers, Leber, Milz, Blut, Pancreas, Thymus, Nieren, Lymphdrüsen, Schilddrüse, Muskeln etc. von Kaltblïtern, Hund, Kaninchen und Rind.

Die Organe waren, soweit angängig, durch Ausspülen nach ibrer Herausnahme von Blut befreit worden, oder sie stammten von Thieren, die durch Verbluten getödtet worden waren.

Es zeigte sich nun zunächst, was bereits friiheren Autoren wie Alexander Schmidt und Schönbein bekannt gewesen, dass die einzelnen Organe verschieden stark auf das $\mathrm{H}_{2} \mathrm{O}_{2}$ einwirkten, dass die zur Zerlegung einer bestimmten Superoxydmenge nöthige Zeit für" sie eine verschieden grosse war, während sie alle das eine gemeinsam hatten, dass die Intensität der Einwirkung sich in den ersten Minuten am grössten erwies und dann langsam bis zum Nachlass der Wirkung absank. Eine völlige Zerlegung des zugesetzten Superoxyds wurde selbst von den bestwirksamen Geweben nicht erzielt, wie die Berechnung des abspaltbaren Sauerstoffs auf Grund der Titration der $\mathrm{H}_{2} \mathrm{O}_{2}$-Lösung mit Permanganat ergab. (Das gleiche ist auch für die $\mathrm{H}_{2} \mathrm{O}_{2}$-Zerlegung durch eine Reihe pflanzlicher und thierischer Fermente, Emulsin, Diastase, wässrige Pankreasauszüge, in denen der Wirkung vielleicht nur 
die jeweils noch vorhandenen Zellreste zu Grunde liegen, von $\mathrm{J}$ a c o b s o h ${ }^{1}$ ) nachgewiesen worden.)

Die wesentlichste Differenz in der Intensitït der $\mathrm{H}_{2} \mathrm{O}_{2}$-Zerlegang durch die verschiedenen Gewebe zeigte sich gerade in den ersten Zeitabschnitten ibrer Einwirkung. Das Nähere ergiebt sich aus den folgenden mehrfach wiederholten Versuchsbeispielen.

Verwendet wurde in allen Versuchen $10 \mathrm{ccm} \mathrm{H}_{2} \mathrm{O}_{2}$-Lösung (von $3.7 \%$ $\mathrm{H}_{2} \mathrm{O}_{2}$ ) mit $40 \mathrm{ccm} \mathrm{H}_{2} \mathrm{O}$ verdünnt.

I a Leber vom Hunde $1 \mathrm{gr}$.

\begin{tabular}{c|c|c}
\hline \hline $\begin{array}{c}\text { Nach } \\
\text { Mi- } \\
\text { nuten }\end{array}$ & $\begin{array}{c}\text { Gelildet } \\
\text { ecm } \mathrm{O}_{2}\end{array}$ & Bemerkungen \\
\hline $\mathbf{1} / 2$ & 50 & Durch starkes Schütteln \\
$\mathbf{1}$ & 55 & am Ende des Ver- \\
$\mathbf{2}$ & 60 & suches tritt noch \\
$\mathbf{3}$ & 62 & Sauerstoff über bis \\
4 & 65 & 97 ccm; nach 24 Std. \\
$\mathbf{6}$ & $661 / 2$ & 97 ccm. \\
7 & 70 & Die zugesetzte $\mathrm{H}_{2} \mathrm{O}_{2}$ - \\
8 & 72 & Lösung bätte bei voll- \\
9 & 75 & ständiger Zerlegung \\
12 & 76 & ergeben müssen 102 \\
14 & $761 / 2$ & ccm. \\
16 & 78 & \multicolumn{2}{|l}{} \\
23 & 80 &
\end{tabular}

II. Pankreas vom Rind $1 \mathrm{gr}$.

\begin{tabular}{c|c}
\hline $\begin{array}{c}\text { Nach } \\
\text { Mi- } \\
\text { nuten }\end{array}$ & ccm $\mathrm{O}_{2}$ gebildet \\
\hline 1 & 38 \\
2 & 58 \\
3 & 67 \\
4 & 71 \\
5 & - \\
6 & 77 \\
7 & 82 \\
13 & Durch starkes Schütteln \\
& am Ende des Ver- \\
& suches werden noch \\
& übergetrieben O $O_{2}$ ccm \\
& bis 98, nach 24 Std. 98.
\end{tabular}

I b. Leber vom Hunde $2 \mathrm{gr}$.

III. Pankreas vom Rind $2 \mathrm{gr}$.

\begin{tabular}{c|c}
\hline $\begin{array}{c}\text { Nach } \\
\text { Mi- } \\
\text { nuten }\end{array}$ & cem $\mathrm{O}_{2}$ gebildet \\
\hline & 76 \\
1 & 80 \\
2 & 81 \\
3 & 82 \\
4 & 84 \\
5 & 84 \\
7 & Nach Stunden \\
& 90
\end{tabular}

\begin{tabular}{c|c}
\hline $\begin{array}{c}\text { Nach } \\
\text { Mi- } \\
\text { nuten }\end{array}$ & cem $0_{2}$ gebildet \\
\hline 1 & 48 \\
2 & 65 \\
3 & 70 \\
4 & 74 \\
5 & 76 \\
6 & 77 \\
7 & 79 \\
8 & -59 \\
11 & Durch Schütteln \\
& bis 98
\end{tabular}

1) Zeitschrift für Physiol. Chemie. Bd. 16. 
Die Beđeut. gewisser Nucleoproteïde für d. oxydat. Leistung der Zelle. 619

IV. Gehirn vom Hunde $2 \mathrm{gr}$.

\begin{tabular}{|c|c|}
\hline $\begin{array}{c}\text { Nach } \\
\text { Minuten }\end{array}$ & $\begin{array}{l}\text { Gebildet } \\
\operatorname{ccm~} \mathrm{O}_{2}\end{array}$ \\
\hline $\begin{array}{r}1 \\
2 \\
3 \\
4 \\
5 \\
6 \\
7 \\
8 \\
9 \\
13 \\
16 \\
23 \\
27 \\
30 \\
35 \\
39 \\
46\end{array}$ & $\begin{array}{l}9 \\
19,6 \\
25 \\
30,8 \\
35 \\
38 \\
42 \\
45,6 \\
49 \\
56,6 \\
61 \\
67 \\
70 \\
71,8 \\
73 \\
74 \\
78\end{array}$ \\
\hline \multicolumn{2}{|c|}{$\begin{array}{c}\text { Durch Schütteln am Ende } \\
\text { des Versuches } 84 .\end{array}$} \\
\hline \multicolumn{2}{|c|}{ V. Blut $1 \mathrm{gr}$ vom Hunde. } \\
\hline $\begin{array}{c}\text { Nach } \\
\text { Minuten }\end{array}$ & $\begin{array}{l}\text { Gebildet } \\
\mathrm{ccm} \mathrm{O}_{2}\end{array}$ \\
\hline $\begin{array}{l}1 \\
2 \\
3 \\
4 \\
5 \\
6 \\
7\end{array}$ & $\begin{array}{l}76 \\
80 \\
81 \\
85 \\
86 \\
88 \\
91\end{array}$ \\
\hline
\end{tabular}

Durch Schütteln werden noch übergetrieben $\mathrm{ccm} \mathrm{O}_{2}$ bis 99 .

VI. Muskeln rom Hunde $2 \mathrm{gr}$.

\begin{tabular}{c|c}
\hline $\begin{array}{c}\text { Nach } \\
\text { Minuten }\end{array}$ & $\begin{array}{c}\text { Gebildet } \\
\text { ccm } 0_{2}\end{array}$ \\
\hline 1 & 8 \\
2 & 13 \\
3 & 18 \\
4 & 22 \\
5 & 27 \\
6 & 32 \\
7 & 36 \\
8 & 40 \\
9 & $\mathbf{4 4}$ \\
10 & $\mathbf{4 6}$ \\
11 & $\mathbf{4 9}$ \\
13 & 54 \\
15 & 56 \\
17 & 60,5
\end{tabular}

Fortsetzung Versuch VI.

\begin{tabular}{c|c}
\hline $\begin{array}{c}\text { Nach } \\
\text { Minuten }\end{array}$ & $\begin{array}{c}\text { Gebildet } \\
\text { ccm } \mathrm{O}_{2}\end{array}$ \\
\hline 19 & 62 \\
23 & 67 \\
31 & 70 \\
37 & 72 \\
60 & 76
\end{tabular}

Nach Beendigung des Versuches durch Schütteln 94.

VII. Eierstock vom Frosch $2 \mathrm{gr}$.

\begin{tabular}{c|c}
\hline $\begin{array}{c}\text { Nach } \\
\text { Minuten }\end{array}$ & $\begin{array}{c}\text { Gebildet } \\
\text { ccm } \mathrm{O}_{2}\end{array}$ \\
\hline 1 & 4 \\
2 & 10 \\
3 & 17 \\
4 & 24 \\
5 & 29 \\
6 & 34 \\
7 & 37 \\
8 & 42 \\
9 & 43 \\
10 & 45 \\
12 & 47 \\
15 & 49 \\
17 & 50,5 \\
18 & 55,5 \\
21 & 66 \\
28 & 71
\end{tabular}

VIII. Eileiter vom Frosch $2 \mathrm{gr}$

\begin{tabular}{c|c}
\hline $\begin{array}{c}\text { Nach } \\
\text { Minuten }\end{array}$ & $\begin{array}{c}\text { Gebildet } \\
\text { ccm } 0_{2}\end{array}$ \\
\hline 1 & 10 \\
2 & 19 \\
3 & 24 \\
4 & 29 \\
5 & 31 \\
6 & 34 \\
8 & 37,6 \\
9 & 39 \\
10 & 40,4 \\
11 & 41,8 \\
15 & 44 \\
18 & 46 \\
25 & 50 \\
28 & 55 \\
35 & 60 \\
38 & 62 \\
50 & 67 \\
55 & 69 \\
61 & 70 \\
54 & 71 \\
77 & 73 \\
92 & 75
\end{tabular}


IX. Thymus vom Kalbe $1 \mathrm{gr}$.

\begin{tabular}{c|c}
\hline $\begin{array}{c}\text { Nach } \\
\text { Minuten }\end{array}$ & $\begin{array}{c}\text { Gebildet } \\
\text { ccm } \mathrm{O}_{2}\end{array}$ \\
\hline 1 & 30 \\
2 & 50 \\
3 & 60 \\
4 & 69 \\
5 & 73 \\
7 & 77 \\
10 & 80 \\
13 & 84
\end{tabular}

X. Milz vom Hunde $1 \mathrm{gr}$.

\begin{tabular}{c|c}
\hline $\begin{array}{c}\text { Nach } \\
\text { Minuten }\end{array}$ & $\begin{array}{c}\text { Gebildet } \\
\text { ccm } 0_{2}\end{array}$ \\
\hline 1 & 76 \\
2 & 80 \\
3 & 81 \\
4 & 82 \\
5 & 83 \\
7 & 84 \\
12 & 84
\end{tabular}

Nach 5 Stunden 90.

Durch Schütteln bis 100 übergetrieben.

ob etwa die jeweiligen Ar. beitsbedingungen, unter denen sich die Gewebe befinden, ihre $0 x y$ dative Kraft beeinflussen, wenigstens soweit dieselbe sich an der Zerlegung des $\mathrm{H}_{2} \mathrm{O}_{2}$ erkennen lässt, wurden einige Versuche mit den Muskeln von Kaltblütern gemacht, die mehrere Stunden hindurch vermittels Strychnin in Tetanus gehalten worden waren. Es ergab sich keine Differenz gegenüber der Leistung nicht tetanisirter Muskeln.

XI. 2 gr Froschmuskel rom Oberschenkel.

\begin{tabular}{c|c}
\hline $\begin{array}{r}\text { Nach } \\
\text { Minuten }\end{array}$ & $\begin{array}{c}\text { Gebildet } \\
\text { cem } \mathrm{O}_{2}\end{array}$ \\
\hline $\mathbf{1}$ & 7 \\
2 & 13,8 \\
3 & 18 \\
4 & 22 \\
5 & 26 \\
$\mathbf{6}$ & 29 \\
$\mathbf{8}$ & 36 \\
9 & 40 \\
10 & 43 \\
$\mathbf{1 1}$ & 46 \\
12 & 48 \\
$\mathbf{1 3}$ & 51 \\
14 & 53 \\
15 & 56 \\
16 & 57,5 \\
17 & 59,5 \\
18 & 61,5 \\
19 & 63 \\
20 & 65 \\
22 & 67,8 \\
24 & 68,6 \\
26 & 70,4 \\
28 & 71 \\
30 & 73 \\
$\mathbf{3 7}$ & 79 \\
&
\end{tabular}

XII. 2 gr Froschmuskel nach 3 stündigem Strychnin-Tetanus.

\begin{tabular}{c|c}
\hline $\begin{array}{c}\text { Nach } \\
\text { Minuten }\end{array}$ & $\begin{array}{c}\text { Gebiliet } \\
\text { ccm } 0_{2}\end{array}$ \\
\hline 1 & 5 \\
2 & 10,5 \\
3 & 14 \\
4 & 19,5 \\
5 & 23 \\
6 & 28,5 \\
7 & 34 \\
8 & 40 \\
9 & 45 \\
10 & 47 \\
11 & 53 \\
12 & 55 \\
13 & 57 \\
14 & 60 \\
15 & 62,5 \\
16 & 64,5 \\
17 & 66 \\
18 & 68 \\
19 & 70 \\
20 & 71,5 \\
22 & 74 \\
24 & 74 \\
26 & 79 \\
28 & 79 \\
30 & 82 \\
32 & 86 \\
&
\end{tabular}

Die gegen den nebenanstehenden Versuch sich ergebende Differenz liegt innerhalb der Breite der individuellen Schwankung. 
Die Bedeut. gewisser Nucleoproteïde für d. oxydat. Leistung der Zelle. 621

Aus den angefuhrten Versuchen ergiebt sich aufs deutlichste, dass sich die einzelnen Gewebe in ibrer oxydativen Energie ganz wesentlich verschieden verhalten, soweit wir die relativ rasch und leicht eintretende Zerlegung von $\mathrm{H}_{2} \mathrm{O}_{2}$ als Maasstab für dieselbe anzusehen vermögen. Wir ersehen, wie zur Abspaltung einer bestimmten Sanerstoffmenge die einzelnen Gewebe verschieden lange Zeit brauchen und erkennen die Verschiedenheit der jeweiligen Zerlegungsintensität auch ganz besonders gerade innerhalb der ersten Zeitabschnitte der ablaufenden Reaction, in denen sie am grössten ist und in verschiedenem Maasse anwächst, während mit der Dauer der Einwirkung und Abnahme der Energie der Zerlegung sich die Differenz verwischt. Wir können mit Zugrundelegung dieser Thatsachen leicht eine Scala aufstellen, die die Gewebe nach der Stärke ihrer Einwirkung geordnet erhält. Dieselbe ergiebt etwa folgende Reibe:

Blut, Milz, Leber, Pankreas, Thymus, Gehirn, Muskeln, Eierstock, Eileiter.

In gleicher Weise haben, wie die mittlerweile erschienenen Arbeiten vou Abelous u. Biarnès ${ }^{1}$, und $S$ alkowski ${ }^{2}$ ) uns zeigen, diese Autoren die Bildung von Salicylsäure aus dem Salicylaldehyd benutzt, um an der Menge der in einer bestimmten Zeit gebildeten Salicylsäure die Intensität der Oxydationsenergie der einzelnen Gewebe zu messen. Die ersteren benutzten ausserdem zu gleichem Zwecke die von uns angegebene Bildung des Indophenols aus farbloser Vorstufe und konnten so durch Vergleich der jeweiligen Intensität der Farbstoffbildung auf colorimetrischem Wege ihre Resultate controlliren ${ }^{3}$ ). In beiden Fällen ergaben sich Reihen, die der von uns aufgestellten fast völlig entsprechen.

Nach A belou s und B i ar nès: Milz, Leber, Lunge, Schilddrüse, Niere, Thymus, Muskeln, Gehirn, Pankreas.

Nach S alk ow k i: Leber, Milz, Niere, Pankreas, Muskeln.

Der Frage, inwieweit die Ergebnisse der Katalyse des $\mathrm{H}_{2} \mathrm{O}_{2}$ abluängig sich erwiesen von der Menge der wirksamen Substanz, wurden nur einige wenige Versuche gewidmet, welche zeigten, dass der Ablauf der Reaction wohl in den ersten Minuten der

1) Archives de Physiolog. normal et path. VII. 1895. VIII. 1896.

2) Archiv f. patholog. Anatomie u. Physiolog. Bd. 147. 1. 1897.

3) Comptes rendus de la Société de Biologie 1896.

E. Pflüger Archiv f. Phgsiologie. Bd. 67. 
Einwirkung eine Beschleunigung erfuhr bei Verwendung doppelter oder grösser Substanzmengen, welche jedoch mit absinkender Energie der Zerlegnng nachliess. Fin bestimmtes Verhältniss zwischen der Intensität der Sauerstoffabspaltung aus $\mathrm{H}_{2} \mathrm{O}_{2}$ und der Menge des verwendeten' Gewebes liess sich nicht construiren. Es dürfte ein Leichtes sein, die Erfahrungen von $M$ edvedew ${ }^{1}$ ) über die Abhängigkeit der Salicylsäurebildung aus dem Aldehyd von der Menge der jeweils wirksamen Substan ${ }^{2}$ auch an der Katalyse des $\mathrm{H}_{2} \mathrm{O}_{2}$ nachzuprüfen.

Immerhin sehen wir, dass die vergleichenden Werthe, wie wir sie für die Energie der $\mathrm{H}_{2} \mathrm{O}_{2}$-Zerlegung erbielten, gut îbereinstimmen mit den Werthen, die von A belous und Biarnès einerseits, Salk $0 \mathrm{wski}$ andererseits nach anderen Methoden als Maass der oxydativen Energie gefunden wurden. Diese Thatsache ist wiederum ein never Grund für uns anzunehmen, dass den in diesen Methoden geprüften oxydativen Processen einheitliche Ur s a chen zu Grunde liegen.

II. Ueber den Einfluss von Protoplasmagiften, schädlichen Temperaturen, Säuren and Alkalien.

Die oxydative Leistung der Gewebe ist, wie an früherer Stelle bereits ausgefiihrt wurde, insofern unerschöpflich, als die kleinste Menge derselben immer von Neuem mit fast ungeschwächter Kraft die zugeführten oxydablen Substanzen zn oxydiren vermag.

Doch wird dieselbe von gewissen äusseren Umständen wesentlich beinflusst.

"Protoplasmagifte" wie Cyankalium und Hydroxylamin schwächen sie, in geeigneter Menge zugesetzt, bis zur völligen Vernichtung.

\section{Versuch Xili.}

$1 \mathrm{gr}$ frische Leber; verrührt mit

$60 \mathrm{ccm}$ Wasser $+5 \mathrm{~cm} 1 \%$ CNKLösung und $10 \mathrm{cem} \mathrm{H}_{2} \mathrm{O}_{2}$.

\begin{tabular}{c|c}
$\begin{array}{c}\text { Nach } \\
\text { Minuten }\end{array}$ & $\begin{array}{c}\text { ccm } \mathrm{O}_{2} \\
\text { gebildet }\end{array}$ \\
\hline 4 & - \\
5 & - \\
7 & 2 \\
10 & 4 \\
15 & 10 \\
$\mathbf{2}$ & 18 \\
35 & 28 \\
nach 3 Tagen & 34
\end{tabular}

1 gr derselben Leber ebenso ohne CNK.

\begin{tabular}{c|c}
\hline $\begin{array}{c}\text { Nach } \\
\text { Minuten }\end{array}$ & $\begin{array}{c}\text { ccm } \mathrm{O}_{2} \\
\text { gebildet }\end{array}$ \\
\hline 4 & 70 \\
5 & 72 \\
7 & 75 \\
10 & 87 \\
15 & 81 \\
22 & 86
\end{tabular}

1) Pflüger's Archiv Bd. 65. 249. 1896. 
Die Bedeut. gewisser Nucleoproteide für d. oxydat. Leistung der Zelle. 623

XIV. $1 \mathrm{gr}$ frische Luber mit $60 \mathrm{ccm}$ Wasser, $2 \mathrm{ccm} 1 \%$ Hydroxylaminlösung und $10 \mathrm{ccm} \mathrm{H}_{2} \mathrm{O}_{2}$

nach 22 Minuten Ireine Spur Sauerstoff gebildet.

XV. $1 \mathrm{gr}$ Pankreas mit $2 \mathrm{~cm} 1 \%$ Hydroxylaminlösung, $60 \mathrm{H}_{2} \mathrm{O}$ und $10 \mathrm{H}_{2} \mathrm{O}_{2}$. nach 1 Stunde keine Spur Sauerstoff gebildet.

Der Einwand, dass eine Zerlegung wohl erfolgt sein könne, der entwickelte Sauerstoff sei aber nur vom stark reducirenden Hydroxylamin in Beschlag genommen worden, ist naturlich binfällig, wenn man die minimalen Mengen $\mathrm{O}_{2}$ bedenkt, die zur Oxydation des verwendeten Hydroxylamins nöthig sind.

Die gleiche deletäre Wirkung hatten diese Gifte auch auf das in den wässrigen Extract der Ġewebe übergehende Princip. Die Katalyse des $\mathrm{H}_{2} \mathrm{O}_{2}$ dureb anorganische Erreger wurde von jenen Giften nicht im entferntesten beeinflusst.

Was den Einfluss der Temperatur betraf, so liess sich im Einklang mit früheren Beobacbtungen constatiren, dass selbst bohe Kältegrade die Einwirkung der Gewebe auf das Superoxyd nicht zu schwächen, geschweige zu zerstören vermochten. Gewebsstiicke, die ich 14 Tage bindurch hatte in einem Eisblock gefrieren lassen, zerlegten aufgethaut, absolnt mit derselben Intensität, wie die eben dem Körper entnommenen. Als Optimum der Temperatur erwies sich die von $30-50^{\circ} \mathrm{C}$, von da ab sank die oxydative Energie stetig, bis sie beim Erhitzen auf etwa $70^{\circ} \mathrm{C}$. fast vollständig und unwiederbringlich verschwand, wenigstens soweit wir die qua n titat i $\mathrm{m}$ ess bare sonst so ausserordentlich leicht und rasch erfolgende Zerlegung von $\mathrm{H}_{2} \mathrm{O}_{2}$ als Maass hierfiir benutzten. Die a uffällige $\mathrm{Thats}$ a ch e konnte hingegen stets beobachtet werden, dass das Gewebe als solehes in $\mathrm{S}$ ubstanz die Fähigkeit in geringstem Maasse (qualitativ nachweisbar) $\mathrm{H}_{2} \mathrm{O}_{2}$ zu zerlegen trotz Erhitzung bis uber die Gerinnung hinaus - selbst bis $120^{\circ}$ - ebenso auch bei Anwesenheit der erwähnten Protoplasmagifte, $\mathrm{n}$ i c h t ganz volls tändig eingebusst hatte, wie sich aus den feinen auftretenden $\mathrm{O}_{2}$-Bläschen respective der Bläuung der mit dem Superoxyd auf das Gewebe gebrachíen Guajactinctur erkennen liess. Allerdings betraf dieses Vermögen - und das ist interessant nur das Gewebe als solches; es war nicht mehr nachweisbar, sobald dasselbe, wie sonst üblich, in $30-50 \mathrm{ccm}$ Wasser aufgeschwemmt untersucht wurde. Ein Leberstuickehen, das bis zur Gerinnung des Eiweisses erhitzt, in Wasser suspendirt und mit $\mathrm{H}_{2} \mathrm{O}_{2}$ 
versetzt, nach Tagen noch kaum eine Spur $\mathrm{O}_{2}$ im Eudiometerrohre erkennen liess und in dieser Vertheilung anch Guajac nicht bläute - zeigte diese Reaction, sobald es mit einigen Tropfen Superoxyd benetzt auf ein mit Guajac getränktes Filtrirpapier gebracht wurde.

Wir müssen diese Reaction als eine für das Gewebe spezifische ansehen, da andere organische Substrate, wie z. B. Eiweisskörper irgend welcher Art u. dgl. sie unter keinen Umständen liefern. Wir wollen diese Fähigkeit der Gewebe, die selbst nach eingreifender Behandlung und nach Verlust der Kraft, $\mathrm{H}_{2} \mathrm{O}_{2}$ in quantitativ grossem Maasse zu zerlegen noch in Action tritt, als Contactwirkung bezeichnen, weil sie ausschliesslich an die ungelöste Substanz gebunden ist und der Lösnng nicht mehr zukommt. Wir werden uns denken müssen, dass der nach Einwirkung hoher Temperaturen und Protaplasmagifte zurückbleibende Rest oxydativer Kraft ein so geringer ist, dass er nur unterstützt durch eine Oberflächenmassenwirkung der einzelnen Molecule, die ja der Lösung abgeht, in Erscheinung treten kann. Halten wir hierbei diese Oberflächenwirkung für wesentlich, so bildet sie, wie gesagt, natürlich nicht allein die Ursache für das Eintreten der von anderen Substraten nicht geleisteten Reaction.

Auf welchem Wege es gelingt, auch diese "Contactwirkung" als letzten Rest der ursprünglichen Kraft zu vernichten, darüber soll an späterer Stelle Näheres mitgetheilt werden.

Noch wesentlich empfindlicher wie gegen hohe Temperaturen verhält sich die oxydative Kraft der Gewebe zugesetzten Säuren und Alkalien gegenüber. Kaustische Alkalien und Mineralsäuren bemmten in ausserordentlich geringer Concentration schon die Zerlegung von $\mathrm{H}_{2} \mathrm{O}_{2}$, hoben sie beziehentlich völlig auf, ohne dass eine nachfolgende Neutralisation die ursprïngliche Wirkang hätte wiederherstellen können. Neutralsalze erwiesen sich als unschädlich. Die blosse Anwesenheit der zugesetzte Säuren resp. Alkalimengen konnte den Process der O-Uebertragung nicht hindern, was sich daraus ergab, dass eine wesentliche Behinderung der $\mathrm{H}_{2} \mathrm{O}_{2}$-Zerlegung ausblieb, wenn jene erst in dem Augenblicke zugefügt wurden, wenn das Gewebe mit der $\mathrm{H}_{2} \mathrm{O}_{2}$-Lösung zusammentraf.

Es trat vielmehr nachweisbare Schädigung erst dann ein, wenn das Gewebe ctwa $3-5$ Minuten mit ihnen grïndlich mecha- 
Die Bedeut. gewisser Nucleoproteïde für d. oxydat. Leistung der Zelle. 625 nisch hearbeitet worden war. Hierbei konnte auch die interessante Beobachtung gemacht werden, dass sehr geringe Alkalimengen sogar eine Steigerung der oxydativen Leistung zur Folge hatten. (Diese Beobachtung correspondirt mit einer analogen von Jac obsson ${ }^{1}$ ) an Emulsin und Pankreasauszügen.)

\section{Versuch XXI.}

$1 \mathrm{gr}$ frischer Leber (vom Hunde) wurde 5 Minnten mit der angegebenen Alkali beziehentlich Säuremenge und Wasser in der Reibschale verrührt; hierauf wurden $10 \mathrm{~cm} \mathrm{H}_{2} \mathrm{O}_{2}$-Lösung in der früher angegebenen Weise zugefügt und das entstehende Sauerstoffgas aufgefangen.

\begin{tabular}{|c|c|c|c|c|c|}
\hline \multicolumn{6}{|c|}{ Cubikcentimeter $\mathrm{O}_{2} \mathrm{gebildet}$} \\
\hline 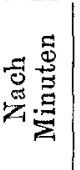 & $\begin{array}{c}1 \mathrm{ccm} 1 / 10 \\
\mathrm{KOH}+49 \\
\mathrm{H}_{2} \mathrm{O}\end{array}$ & $\begin{array}{c}2 \mathrm{ccm} 1 / 10 \\
\mathrm{KOH}+48 \\
\mathrm{H}_{2} \mathrm{O}\end{array}$ & $\begin{array}{l}\text { bei Zusatz vor } \\
\begin{array}{c}3 \mathrm{cem} 1 / 10 \mathrm{KOH} \\
+47 \mathrm{H}_{2} \mathrm{O}\end{array}\end{array}$ & $\left|\begin{array}{c}5 \mathrm{ccm} 1 / 10 \mathrm{KOH} \\
+45 \mathrm{H}_{2} \mathrm{O}\end{array}\right|$ & $\begin{array}{c}10 \mathrm{ccm}^{1 / 10} \\
\mathrm{KOH} \\
+40 \mathrm{H}_{2} \mathrm{O}\end{array}$ \\
\hline $\begin{array}{r}2 \mathrm{~h} \\
1 \\
2 \\
3 \\
4 \\
5 \\
6 \\
7 \\
8 \\
9 \\
10 \\
11 \\
12 \\
13 \\
14 \\
15 \\
16\end{array}$ & \begin{tabular}{|c|c|c|}
84 \\
86 \\
$86^{1} / 2$ \\
- \\
$-\overline{7} \mathbf{1} / 2$ \\
- \\
- \\
- \\
- \\
- \\
- \\
- \\
88 \\
Std. nach \\
Beginn des \\
Versuches \\
88
\end{tabular} & $\begin{array}{c}\overline{47} \\
64 \\
73 \\
79 \\
-\overline{84} \\
86 \\
86 \\
- \\
- \\
- \\
89,4 \\
- \\
- \\
91,6 \\
\text { Um } 12 \mathrm{~h} 36 \mathrm{~m} \\
96 \\
\text { nach } 2 \text { Std. } \\
96\end{array}$ & $\left\{\begin{array}{l}\int^{3} \\
\text { Keine Entwick- } \\
\text { lung; ; ach } 2 \text { Std } \\
\text { neutralisirt mit } \\
3 \text { cem } 1 / 10 \mathrm{H}_{2} \mathrm{SO}_{4} \text {; } \\
\text { keine Entwick- } \\
\text { lung; nach 3Std. } \\
\text { ebenfalls nicht. }\end{array}\right.$ & $\mid \begin{array}{l}\text { Keine Entwick- } \\
\text { lung,neutralisirt } \\
\text { mit 5 ccm 1/10 } \\
\mathrm{H}_{3} \mathrm{SO}_{4} ; \text { keine } \\
\text { Entwicklung; } \\
\text { nach 3 Std. eben- } \\
\text { falls nicht. }\end{array}$ & 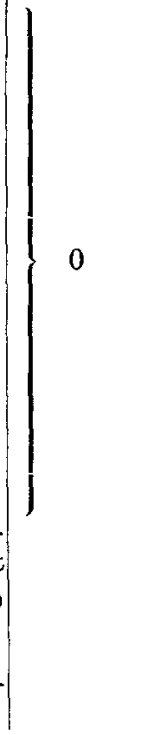 \\
\hline
\end{tabular}

1) a. a. 0 . 


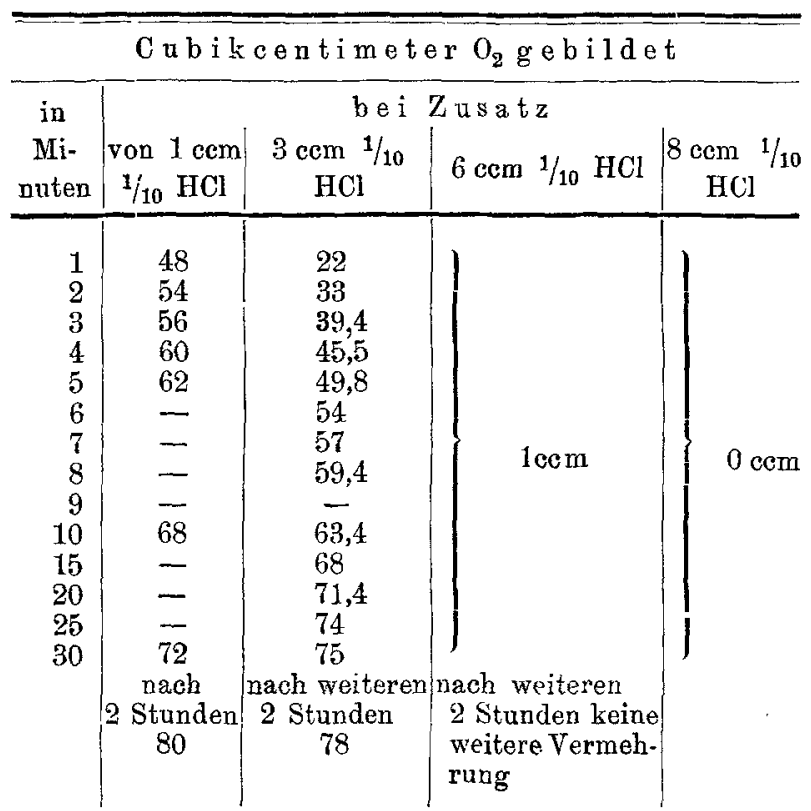

Wenn wir uns bis jetzt $\mathrm{n} \mathfrak{u}$ an die Einwirkung auf $\mathrm{H}_{2} \mathrm{O}_{2}$ gehalten haben, so gesebah dies, weil dasselbe als ausserordentlich bequemes Reagens eine sehr leichte Prüfung der angezogenen Verhältnisse gestattete. Es ist nicht überflussig zu bemerken, dass die Gesetze, welche wir für die 0-Abspaltung aus dem (den Sauerstoff leicht abgebenden) Wasserstoffsuperoxyd festgestellt haben, auch für die Spaltung des Sauerstoffmoleculs sich als gültig erwiesen. Es zeigte sich, dass die oxydative Bildung der erwähnten Farbstoffe, der Indophenole, des Toluylen-Blaus, der Eurrhodine und ebenso die Oxydation des Salicylaldebyds zu Salicylsäure etc. von den gleichen Bedingungen abhängig ist. Der Gehalt einer Organaufschwemmung an Säure oder Alkali, welche eine wesentliche Zerlegung des $\mathrm{H}_{2} \mathrm{O}_{2}$ nicht mebr zuliess, verhinderte ebenso sofort jene erwähnten Farbstoffsynthesen, die Bildung der Salicylsäure etc. ${ }^{1}$ ).

Die bereits erörterte "Contactwirkung." trat bei Verwendung jener anderen schwerer oxydablen Substanzen nicht mehr in Er-

1) Auch Abelous und Biarnès heben hervor, wie ausserordentlich leicht die Bildung der Salicylsäure durch Gewebe bei einem geringen Gehalt an Säure oder Alkali geschädigt, beziehentlich sogar aufgehoben wird. 
Die Bedeut. gewisser Nucleoproteïde für d. oxyảat. Leistung der Zelle. 627

scheinung. Ein erhitzter Leberbrei z. B., der anf Papier ausgestrichen and mit den Indophenolbildnern ïbergossen wurde, zeigte keine Indophenolbildung mehr. $\mathrm{Er}$ zerlegte aber noch immerhin soviel Superoxyd, dass mit Hülfe der gebildeten geringen O-Menge die Vorstufen des Indophenols in dieses übergeführt werden konnten. Die so äusserst geringe Intensität jener "Contactwirkung" reicht wohl aus, das so sehr leicht zerlegibare $\mathrm{H}_{2} \mathrm{O}_{2}$ zu katalysiren, genügt aber nicht mehr zur Spaltung eines 0 -Moleculs, wie es für die Bildung der Indophenole nöthig ist, eine Anschauung, die der an frïherer Stelle von uns ausgesprochenen entspricht.

Unsere Beobachtungen iber jene Farbstoffsynthesen durch organische Gewebe sind im vollen Umfange von Abelous und B i a r n ès bestätigt worden, die die Farbstoffbildung zu einem Vergleich der oxydativen Energie der einzelnen Gewebe benützen. Ihre auf colorimetrischem Wege erzielten Resultate ergaben genan dieselben Vergleichswerthe wie ihre früheren mit Salicylaldehyd und meine mit $\mathrm{H}_{2} \mathrm{O}_{2}$ angestellten Versuche ${ }^{1}$ ). Auch mit Pflanzenzellen, den wässrigen Auszügen verschiedener Pilze hat $\mathrm{B}$ o u $\mathrm{r}$ $q u$ elot ${ }^{2}$ ) die gleichen Synthesen erhalten. Nur Pobla) hat auffälliger Wuise die Bildung der Indophenole durch Organextracte (der Leber) nicht immer erzielt. Woran dieses liegt, geht aus seinen Versuchen nicht ohne Weiteres hervor. Nach meinen umfassenden Erfahrungen ist dieselbe bei ausgebluteten frischen Organen unter allen Umständen zu beobachten. Man muss nur darauf achten, das Blut sorgfältig aus den Organen zu entfernen, da dasselbe das Zustandekommen der Reaction unter Umständen stören kann. Es empfiehlt sich auch, um einen ausgiebigen Contact der Zellen mit der zu oxydirenden Flüssigkeit und dem Sauerstoff zu ermöglichen, ausschliesslich einen von Bindegewebe und Blutgefässen befreiten Zellbrei für diese Versuche anzuwenden.

Dagegen hat $\mathrm{P}$ oh $\mathrm{l}$ ebenfalls durch Pflanzenextracte, die Blätter von Sambucus nigra, Syringa vulgaris, mit Tannennadeln, Amygdalin resp. deren wässrigen Auszïgen die Indophenol-Synthese sehr leicht und intensiv erzielen können. Pohl schliesst nun aus der Thatsache, dass ein Organbrei keine Indophenol-Bläunng liefert, der sogar

1) a. a. 0 .

2) Comptes rendus de la Société de Biologie 1896.

3) Archiv für experimentelle Pathologie Bd. 38. 65. 1896. 
die schwerer eintretende Oxydation des Formaldehyds zu Ameisensäure fertig bringt, während andererseits durch pflanzliche Substrate letztere Reaction überhaupt nicht, erstere dagegen sehr leicht zu erhalten war, darauf, dass die Farbstoffsynthesen nicht als allgemein gültiges Maass der oxydativen Potenz angesehen werden können. Er meint, dass es sich bei der Indophenolreaction einerseits und bei der Oxydation eines Aldehyds andererseits um zwei ganz verschiedene Vorgänge handelte. In dem einen Falle tritt die Oxydation ein, durch den Austritt von zwei Wasserstoffatomen aus zwei Verbindungen, wie die Gleichung zeigt, und ein Zusammentreten der beiden Componenten zu einer nenen Verbindung, also um eine oxydative Synthese unter Wasseraustritt, in dem zweiten, um den einfachen Eintritt eines Sauerstoffatoms in eine COH-Gruppe. Der erstere Vorgang könne nicht gut einen Indicator für den zweiten anders gearteten abgeben.

Abgesehen davon, dass wir zur Zeit die Thatsache bestreiten, dass ein die Oxydation eines Aldehyds lieferndes Substrat die Indophenol-Synthese zu erzengen nicht im Stande sei, können wir die Anschaung $\mathrm{Pohl}$ 's nicht ohne Weiteres acceptiren. Wir sind nicht in der Lage anzunehmen, dass eine oxydative Synthese unter Wasserstoffaustritt in ihrem Wesen eine $\mathrm{p}$ rin z i p i e 11 verschiedene oxydative Leistung repräsentire gegenüber dem Eintritt eines Sauerstoffatoms in ein Molecïl. Wir haben keine Veranlassung von unserer Vorstellung abzugehen, dass das Gewebe in dem einen wie in dem andern Falle die Oxydation bedingt durch eine Spaltung molecularen Sauerstoffs der Luft. In beiden Fällen erfolgt dieselbe durch die Einwirkung des Sanerstofferregers (gewisse in den Geweben enthaltene Substanzen s. u.) und des, beziehentlich cer Sauerstoffnehmer auf molecularen Sauerstoff. Ob Wasserstoff aus dem Sauerstoffnehmer austritt oder Sauerstoff in denselben eintritt, ist für das Princip gleichgiltig. Wenn wir Pohl folgten, dürfte die.Oxydation des Benzylalcohols zu Benzoesäure auch nicht zum Maass der oxydativen Energie benutzt werden, da hier ebenfalls die Oxydation unter Austritt von Wasserstoff erfolgt.

Nach dem, was wir früher ausgeführt haben ${ }^{1}$ ), ist es leicht verständlich, wenn bei der Prüfung der oxydativen Energie des einen Sauerstofferregers eine Oxydation ausbleibt, die bei der

1) Pflüg. Arch. 60. 323. 1895. 
Die Bedeut. gewisser Nucleoproteide für d. oxydat. Leistung der Zelle.

Prtifung eines anderen eintritt. Dann handelt es sich um eine Verschiedenheit in der Kraft der Erreger. Es kann also auch bei der Anwendung desselben Erregers der eine Körper oxydirt werden, der andere nicht. Dies beruht auf der Verschiedenheit der Sauerstoffnehmer. Wenn ein Pflanzenzellenextract Indophenol bildet, aber einen Aldehyd nicht oxydirt, so beweist dies nur, dass seine oxydative Kraft für diese eben schwerer auszuführende Reaction nicht ausreicht, ebenso, wie ein Gewebe, welches diese zu leisten im Stande ist, $\mathrm{H}_{2} \mathrm{O}_{2}$ sicherlich zerlegen wird, nicht aber umgekebrt. Substrate, welche die Indophenol-Synthese liefern, werden also sicherlich in Bezug anf ihre oxydative Energie mit einander nach der Intensität der erzielten Farbstoffbildnng gut verglichen werden können, wie dies $A b e l o u s$ und B i a rn ès gethan haben. Und dass es sich hierbei wenigstens für die thierischen Gewebe nicht um etwas principiell Verschiedenes handelt gegenüber der Bildung der Salicylsäure 'z. B. aus dem Aldehyd, geht ja eben auch daraus hervor, dass die Energiescala der Gewebe für beide Leistungen die gleiche ist.

\section{Ueber die in Wasser löslicben Sauerstofferreger.}

Es war bereits allen Autoren, die sich mit der Frage der Oxydation durch todte Substrate animalischer oder pflanzlicher Natur beschäftigt haben, bekannt, dass das wirksame von den meisten als "Oxydationsferment" bezeichnete Prinzip den Geweben durch Wasser oder verdiunnte Kocbsalzlösungen entzogen werden kann. Wir vermochten das Gleiche nur zu bestätigen. Dass bei dieser Extraction keinerlei wesentliche Veränderung mit dem oxydirenden Agens vor sich ging, erschien schon daraus wahrscheinlich, dass die Wirkung der filtrirten wässrigen Organausziige von den gleichen Bedingungen abhängig war, wie die Action der ursprtunglichen Gewebe vor der Extraction. Die beziiglichen wässrigen Extracte zeigten genau dieselbe Intensität der $\mathrm{H}_{2} \mathrm{O}_{2}$-Zerlegung, sie waren unter einander in Bezug auf die Geschwindigkeit derselben ebenso verschieden wie die ursprünglichen Gewebe und wurden durch die genau gleichen Mengen bestimmter Mineralsäuren und kaustischer Alkalien, durch Protoplasmagifte etc. in der gleichen Weise geschädigt.

Wurde der wässrige Auszug eines Leberzellbreis bis zur Gerinnung erhitzt, so war weder der entstehende Niederschlag noch 
das Filtrat im Stande, irgend welche grössere nachweisbare Sauerstoffmengen aus dem Superoxyd zu entwickeln, doch zeigte der Niederschlag, jedoch nur dieser, kraft der gleichen Contactwirkung, wie wir sie oben von dem Gewebe in toto kennen gelernt haben, die Fähigkeit, durch Zerlegung einer minimalen Menge $\mathrm{H}_{2} \mathrm{O}_{2}$ die Guajactinctur zu bläuen.

Es fragte sich nun, in wie weit man im Stande war, den Geweben durch Wasserextraction die wirksame Substanz zu entziehen. Dies gelang vollkommen nicht. Während bei wiederholter Extraction die Auszüge immer schwächere Wirksamkeit zeigten, ohne dass sie selbst im letzten vollkommen fehlte - zeigte sich doch der sechste Auszug eines Leberzellbreis trotz je 24 stündiger Einwirkung noch als wirksam -, so verblieb dem Organbrei noch die genau gleich intensive oxydative Kraft in Bezug auf die Katalyse von $\mathrm{H}_{2} \mathrm{O}_{2}$, die Oxydation des Salicylaldehyds, die Bildung der Farbstoffsynthesen etc., wie er sie vor der Extraction gehabt hatte.

$$
\mathrm{Versuch} \text { : }
$$

Leberzellenbrei wird sechsmal mit Wasser durch je 24 Stunden extrahirt. - $1 \mathrm{gr}$ der extrahirten Masse werden mit 50 Wasser und $10 \mathrm{~cm} \mathrm{H}_{2} \mathrm{O}_{2}$ gemischt

$\begin{array}{lcccccc}\text { nach Minuten } & 1 & 2 & 3 & 4 & 6 & 8 \\ \text { ccm } \mathrm{O}_{2} \text { entwickelt } & \mathbf{1 2} & \mathbf{7 8 , 4} & 81 & 85 & 88 & 90\end{array}$

Versuch abgebrochen.

Aus dieser wiederholt constatirten Beobachtung ging unzweidentig hervor, dass an der oxydativen Leistung der Gewebe ausser einer in Wasser relativ leicht löslichen Substanz eine in Wasser resp. verdünnten Kochsalzlösungen unlösliche betheiligt sein musste. Die gleichen Beobachtungen hatte für die Katalyse des $\mathrm{H}_{2} \mathrm{O}_{2}$ schon Alexander Schmidt ${ }^{1}$ ) an einer Reibe von Organen gemacht. Für die glycolytische Function des Blutes war von Lépine, Arthas und mir das Gleiche nachgewiesen worden.

Da eingreifendere Methoden als die Extraction mit Wasser, wegen ihres deletären Einflusses auf die oxydative Kraft nicht zu verwenden waren - auch die Behandlung mit Alkohol erwies sich nicht als gleichgïltg (vgl. Salkowski) 一, so wollen wir uns in dieser Arbeit zunächst nur mit dem in Wasser löslichen Princip befassen und die Frage vor der Hand ruhen lassen, ob

1) Zur Blutlehre, Leipzig 1892. 
Die Bedeut. gewisser Nucleoproteïde für d. oxydat. Leistung der Zelle. 631

das im erschöpften Zellenrest noch wirksame Substrat mit dem ersteren verwandt resp. identisch ist oder nicht. Sicher ist das eine, dass für die oxydative Leistung desselben die gleichen Gesetze gelten, wie für das in Wasser lösliche.

Es lag nunmebr die Aufgabe vor, dieses letztere genauer zu studiren resp. isolirt berzustellen. An früherer Stelle schon batten wir uns gegen die ja sonst sehr bequeme Annahme eines oxydativen „Ferments" ausgesprochen. Würde man hierunter, wie früher üblich, ganz allgemein organische Substanzen unbekannter Herkunft und Zusammensetzung verstehen wollen, die in geringster Menge, ohne sich während ihrer Einwirkung irgend wie zn verändern, Umsetzungen irgend welcher Art in organischen Substraten hervorrufen, so wäre ja gegen eine solche Bezeichnung nichts einzuwenden; doch bätte dieselbe nur solange zu gelten, bis eine bessere und zusagendere vorliegt. Ein Ferment im heutigen Sinne, ein "Enzym ", ist natïrlich unser wirksames Prinzip nicht, da wir unter einem solchen nur organische Substanzen verstehen, die unter Aufnahme von Wasser complicirte Verbindungen in einfachere überführen. Das trifft selbstrerständlich für die erwähnten Oxydationen nicht zu.

$\mathrm{Da}$ wir aus früheren Untersuchungen schon darüber unterrichtet sind, dass ausschliesslich die Gewebszellen die oxydative Leistung der Gewebe bedingen und es andererseits kein zellenbaltiges Gewebe des thierischen Organismus giebt, das nicht Oxydationskraft besässe, sei es auch nur, dass diese sich in der Zerlegung von $\mathrm{H}_{2} \mathrm{O}_{2}$ documentirt, so musste natürlich an Substanzen gedacht werden, die allen Zellen des Thierkörpers gemeinsam sind. Da ferner alle sonst näher bekannten Eiweisskörper, die in Frage bätten kommen können, sich selbst schon für die Zerlegung von $\mathrm{H}_{2} \mathrm{O}_{2}$ als unwirksam erwiesen und die Annahme, dass gerade allen Zellen des thierischen und pflanzlichen Organismus ein so mächtig wirkendes "Ferment" als unwesentlicher und nicht zu ihnen direct gehöriger Bestandtheil gewissermaassen nur anhaften sollte, nicht gerade sehr wahrscheinlich erschien, so lag in der That nichts näher als an Verbindungen zu denken, die durch ihre Betheiligung an dem Aufban des für das Leben der Zelle so wichtigen Zellkerns auch für eine wesentliche chemische Leistung der Zelle als ganz besonders geeignet in Betracht kommen konnten. Wir meinen hiermit Verbindungen aus der Gruppe der Nucleoproteïde. Die nähere Veranlassung, dieselben zu prïfen, lag für uns darin, dass von dem 
Nucleoproteïd der Thymus, mit dem sich Lilienfeld beschäftigt hatte, dessen Einwirkung auf $\mathrm{H}_{2} \mathrm{O}_{2}$ bereits bekannt war.

IV. Nueleoproteïde als Sauerstoffüberträger.

1. Ein Nucleoproteïd (Nucleohiston) der Leber.

Als maximal wirksames Organ wurde zunächst die Leber in Angriff genommen. Die dem eben getödteten Thiere (Hund) entnommene Leber wurde von der Pfortader aus durch die Wasserleitung so lange durchgespült, bis die abfliessende Flitssigkeit sich fast frei von Blutfarbstoff erwies, gründlich in der Fleischmühle zerquetscht nnd durch ein mehrfaches Gazefilter gepresst. Der durchtretende Zellbrei wurde mit dem 5 fachen Volumen destillirten Wasser versetzt, des öfteren gut umgerührt nnd 24 Stunden hindurch bei niederer Temperatur stehen gelassen. Der opalescente Wasserextract wurde hierauf vorsichtig von dem Zellbrei abgehoben und durch Leinwand filtrirt. Die von Leberzellen, wie die mikroskopische Untersuchnng lehrte, freie Flïssigkeit wurde nunmehr tropfenweise mit einer sehr verdünnten ( $1 / 10$ Normal-) Salzsäure oder Essigsäure versetzt, bis keine Ausfällung mehr erfolgte und die vorher opalescente Flüssigkeit ganz klar wurde. Die vollständige A usfällnng zu erzielen erschien insofern nicht ganz leicht, als die Fällung schon bei geringem Ueberschuss der zugesetzten Säure sich wieder zu lösen begann. Ausserordentlich erleichtert wurde die Procedur jedoch durch die Benutzung des Lacmoids als Indicator; hatte ich soviel Säure zugesetzt, dass blaues Lacmoid gerade eine Spur Ueberschuss an Säure anzeigte, so konnte die Fällung als vollständig angesehen werden. Das Filtrat ergab dann weder auf Säure- noch auf Alcalizusatz eine Tribung.

Das gut ausgefällte Präcipitat setzte sich relativ rasch $a b$, wurde durch Decantiren und Filtriren auf dem Filter gesammelt, mit Wasser gewaschen, zur Reinigung nochmals in einer entsprechenden Menge $1 / 1_{0}$ Normalsodalösung gelöst, wiederum gefällt, auf einem Filter gesammelt und frisch untersucht.

Die graubraune Substanz zeigte, feucht untersucht, folgende Eigenschaften:

Sie zerlegte in ausgefälltem Zustande sowohl wie in sehr verdiunnter Sodalösung gelöst schon in geringster Menge die $S$ u p e roxydlös ung und bläute dadurch die Guajactinctur.

Die Zerlegung des $\mathrm{H}_{2} \mathrm{O}_{2}$ erfolgte annähernd mit derselben Intensität, wie von Seiten der ursprünglichen Leber selbst. 
Die Bedeut. gewisser Nucleoproteìde für d. oxydat. Leistung der Zelle. 633

$$
\text { Y Versuch. }
$$

$0,2 \mathrm{gr}$ der frischen Substanz werden in $50 \mathrm{ccm}$ Wasser unter Zusatz von $4 \mathrm{ccm} 1 / 10$ Normalsodalösung gelöst und mit $10 \mathrm{ccm} \mathrm{H}_{2} \mathrm{O}_{2}$-Lösung $(3,4 \%)$ vermischt.

$\begin{array}{lcccccccc}\text { Nach Minuten } & 1 & \mathbf{2} & \mathbf{3} & \mathbf{5} & 6 & 13 & 17 & 25 \\ \text { ccm } \mathrm{O}_{2} \text { entwickelt } & 40 & 55 & 65 & 70 & 73 & 75 & 82 & 90\end{array}$

Versuch beendet nach 24 Stunden 90.

$0,2 \mathrm{gr}$ der frischen Substanz in $50 \mathrm{ccm}$ Wasser suspendirt mit $10 \mathrm{ccm}$ $\mathrm{H}_{2} \mathrm{O}_{2}$ vermischt.

$\begin{array}{lcccccccc}\text { Nach Minuten } & 1 & 2 & 3 & 4 & 5 & 8 & 9 & 16 \\ \text { cem } O_{2} \text { entwickelt } & 19 & 28 & 41 & 55 & 69 & 85 & 87 & 95\end{array}$

Säuren, Alkalien, höhere Temperaturen und Protoplasmagifte wirkten auf unsere Substanz genau in demselben Umfange schädigend beziehentlich vernichtend ein, wie auf die Leber iiberhaupt.

Versuch.

$0,2 \mathrm{gr}$ frischer Substanz, gelöst in $50 \mathrm{ccm}$ Wasser und $5 \mathrm{ccm} \mathrm{1/10}$ Normal $\mathrm{KOH}$ zerlegen $\mathrm{H}_{2} \mathrm{O}_{2}$ innerhalb. 24 Stunden überhaupt nicht nachweisbar. Bei erfolgter Neutralisation mit $5 \mathrm{~cm} 1 / 10$ Normal $\mathrm{H}_{2} \mathrm{SO}_{4}$ erfolgte die Zerlegung ebenfalls nicht mehr. In gleicher Weise vcrnichtete ein Gehalt von $5 \mathrm{~cm}$ $1 / 10 \mathrm{H}_{2} \mathrm{SO}_{4}$ in $50 \mathrm{ccm}$ W'asser die katalytische Kraft unseres Substrates bei dem gleichen Mengenverhältniss derselben. Wurden $0,2 \mathrm{gr}$ der frisch gefällten Verbindung in Sodalösung im Ueberschuss gelöst, so erwies sich bei einem Gehalt von $12 \mathrm{~cm} 1 / 10$ Normalsoda in $50 \mathrm{ccm}$ Wasser die Katalyse des Superoxyds bereits erheblich geschwächt.

$$
\begin{array}{lcccccc}
\text { Nach Minuten } & 1 & 2 & 4 & 8 & 14 & 24 \\
\text { cem } \mathrm{O}_{2} \text { entwickelt } & 8 & 19 & 24 & 43 & 60 & 80 \text { u. s. w. }
\end{array}
$$

Wurde unsere Substanz, deren Gerinnnung bei $65^{\circ} \mathrm{C}$. erfolgte, auf $90-100^{\circ} \mathrm{C}$ erhitzt, so verlor sie bei nachheriger Lösung in Soda oder Aufschwemmung in Wasser die Fähigkeit, irgendwie nachweisbare Mengen $O_{2}$ aus dem Superoxyd zu entbinden. Es wurden innerlialb 24 Stunden etwa nur $1 \mathrm{~cm} \mathrm{O}_{2}$ gemessen. Doch vermochte die Substanz auch in coagulirtem Zustande eine qualitativ nachweisbare Spur $\mathrm{H}_{2} \mathrm{O}_{2}$ zu katalysiren, wie die Guajacbläuung bei Anwesenheit von $\mathrm{H}_{2} Q_{2}$ erwies. In gleicher Weise vermochten CNH und Hydroxylamin wohl der gelösten Substanz jede katalysirende Fähigkeit zu rauben, nicht aber diese „Contactwirkung ${ }^{\text {zu }}$ vernichten.

Die mit Alkohol aus neutraler Lösung gefällte Substanz hatte ihre katalytische Kraft behalten, wenn sie allerdings auch schwächer 
war, wie vorber. Nach mebr als 2-3 tägiger Behandlung mit Alkohol verschwand sie allerdings fast völlig. Der frischen Alkoholfällung liess sie sich durcb Behandlung mit Wasser entziehen; der wässrige Auszug wirkte wiederum katalytisch.

Bei der Prüfung der Einwirkung unserer Substanz auf die erwähnten $\mathrm{F}$ a r b s t of $\mathrm{s}$ y $\mathrm{u}$ thes en wurde in der bereits früher erwähnten Weise verfahren.

Die frisch ansgefällte Substanz wurde in dünner Scbicht in Form von Buchstaben auf Filtrirpapier ausgestrichen und mit den bezuiglichen Farbstoffvorstufen übergossen. Es zeigte sich, dass in allerkürzester Zeit auf der Substanz Farbstoffbildung eintrat, während die übrigen Partien des Filtrirpapiers erst wesentlicb später Färbung zeigten. Es wurde innerbalb kurzer Zeit erzielt:

a) Bildung von Indophenol aus $\alpha$-Naphtol, Soda und Paraphenylendiamin.

B) von Toluylenblau aus Paraphenylendiamin und Methatoluylendiamin.

r) von Bindschedler's Grün aus Dimethylanilin und Dimethylparaphenylendiamin.

d) Oxydation des Dimethylparapbenylendiamins (Reaction von W u r s ter).

Die synthetische Farbstoffhildung. blieb aus bei Anwesenheit von Blausäure, Hydroxylamin, sie fehlte anch dem enagulirten und auf $70-80^{\circ}$ erhitzten Präparate. Bei Behandlung der frischen Fällung mit Mineralsäuren und starken Alkalien in einer Concentration, die bereits ansgiebige $\mathrm{H}_{2} \mathrm{O}_{2}$-Zerlegung verhinderte, wurde die Bescbleunigung der Farbstoffbildung ebenfalls vermisst. Dass es sich hierbei nicht um eine Behinderung der Farbstoffbildung als solcher, sondern in der That um eine direkte schädigende Einwirkung auf die O-übertragende Kraft unserer Substanz handelte, ging zunächst daraus hervor, dass die Farbstoffbildung, soweit sie eben spontan erfolgte, nicht ausblieb; die mit unserer Substanz beladenen Theile des Filtrirpapiers wurden nur nicht friber gefärbt als die übrigen. Das Gleiche bewies auch die Thatsache, dass der Zusatz von wenigen Tropfen concentrirter $\mathrm{KOH}$ zu einer verdünnten Lösung der Vorstufe des Toluylenblaus die spontan erfolgende Synthese derselben erheblich beschleunigte, jedoch die Beschleunigung durch unsere Substanz völlig verhinderte. 
Die Bedeut. gewisser Nucleoproteide für đ. nxydat. Leistung der Zelle. 635

Sehr geringe Mengen von kaustischen Alkalien und Ammoniak verbinderten die Beschleunigung der Farbstoffbildung schon in einer Concentration, die selbst noch $\mathrm{H}_{2} \mathrm{O}_{2}$-Zerlegung gestattete. Die erstere erfolgte dann rasch nach nachträglicher Zufügung von $\mathrm{H}_{2} \mathrm{O}_{2}$. - Daraus ging hervor, dass die Fähigkeit, aus dem O-Molecul $\mathrm{O}$ zu activiren, leichter geschädigt wird, als die, es dem $\mathrm{H}_{2} \mathrm{O}_{2} \mathbf{z u}$ entziehen.

Aus dieser ausserordentlichen Empfindlichkeit unserer Verbindung lässt sich erschliessen, wie von der sorgfältigen Herstellung derselben, bezw. dem Vermeiden eines jeden Säureüberschusses bei der Ausfällung das Gelingen der erwähnten Versuche abhängig ist.

Schliesslich wurde noch geprüft, ob auch die Fähigkeit Aldebyde zu oxydiren, aus dem Salicylaldehyd die Salicylsäure zu bilden, unserem Präparate zu eigen war. Hierbei verfuhr ich in folgender Weise: Der Wasserauszug von etwa $500 \mathrm{gr}$ Leber wurde mit Säure gefällt; die frische Fällung, wurde vorsichtig unter Zusatz von $1 / 10$ Normalsodalösung in Wasser gelöst, auf 1 Liter aufgefiullt, nach Zusatz von $2 \mathrm{ccm}$ Salicylaldehyd anf dem Wasserbade bei $45-50^{\circ} \mathrm{C}$. durch 24 Stunden belassen, während welcher Zeit ein continuirlicher Luftstrom mit der Wasserstrahlpumpe durchgeleitet wurde. Nach Ausfällung mit $1 / 10$ norm. $\mathrm{H}_{2} \mathrm{SO}_{4}$ wurde filtrirt, bei stark alkal. Reaction eingedampft, mit Alkohol mehrfach extrahirt, der nach Verdunsten desselben restirende Rïckstand mit $\mathrm{HCl}$ stark angesäuert und mit Aether mebrfach extrahirt. Zur Entfernung etwa noch vorhandenen Salicylaldehyds wurden die vereinigten ätherischen Auszüge mit Natriumbisulfitlösung, dann mit destillirtem Wasser gewaschen und schliesslich der Aether verdunstet. Die beim Erkalten desselben zurückbleibenden weissen langen Nadeln wurden in etwas Alkohol gelöst, mit Wasser zu $50 \mathrm{ccm}$ aufgefüllt und unter Verwendung von Phenolphtaleïn mit $1 / 20$ normal. $\mathrm{NaOH}$ titrirt. Controlversuche hatten gezeigt, dass bei diesem Verfahren jede Spur nicht oxydirten Aldehyds entfernt wurde. És konnte somit auch der positive Ausfall der Eisenchloridreaction auf gebildete Salicylsäure bezogen werden.

I. Versuch. In 24 Stunden lieferte Rindsleber $41,4 \mathrm{mgr}$ Salicylsäure.

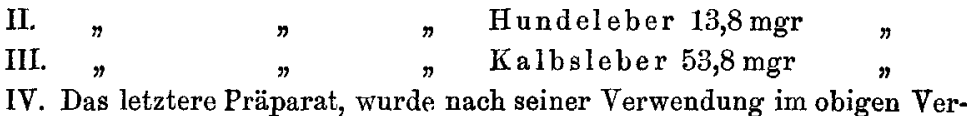
suche gefällt und 14 Tage hindurch in einem Eisblock gefroren vor dem 
Fenster gehalten; nach dem Aufthauen za einem Versuche von 24 stündiger Dauer verwendet, ergab dasselbe noch $18 \mathrm{mgr}$ Salicylsäure.

Die gewonnenen Salicylsänremengen sind nicht viel geringer als die mit den Gesammtwasseranszigen der Leber resp. dem suspendirten Leberbrei von anderen Autoren erhaltenen.

In Bezug auf Temperatur und das Verbalten zu Säuren und Alkalien lagen hier ungefäbr die gleichen Verhältnisse vor, wie sie bereits bei der Einwirkung auf $\mathrm{H}_{2} \mathrm{O}_{2}$ und die besprochenen Farbstoffbildungen berichtet warden.

So zeigte ein Versuch, in welchem unser Präparat durch 20 Minuten bis 'zur Gerinnung erhitzt worden war, keine SalicylsäureKrystalle und eine Accidität von 0,5 1/10 normal Alkali, während die Eisenchloridreaction positiv ausfiel. Würde selbst die ganze Acidität auf Salicylsänre bezogen, so hätte dieselbe auch nur $6,9 \mathrm{mg}$ derselben entsprochen. In einem anderen Versuche, in welchem unser Präparat durch Ausfällung mit zu starker Säure gewonnen worden war - es war das zweifache der nothwendigen Menge zugesetzt worden - , ergab in gleicher Weise mit Salicylaldehyd verarbeitet eine Acidität yon $0,1 \mathrm{ccm} / 1 / 10$ normal Alkali and keine Spur Eisenchloridreaction.

Nachdem aus den angeführten Versucben sich mit der allergrössten Deutlichkeit gezeigt hatte, dass das durch Säure gefällte Präparat dieselben Oxydationen auszuführen vermochte, wie das urspringliche Gewebe selbst und sein wässriger Auszug und dass dasselbe auch denselben Bedingungen in seiner Leistungsfähigkeit unterworfen war, wie jene, so konnte es natürlich keinem Zweifel unterliegen, dass unsere Substanz das oxydationserregende Princip der Leberzelle (oder mindestens eines derselben) repräsentiren musste.

Da die wässrigen Gewebsauszüge nach vollständiger Ausfällung unseres Präparates in ihrem Filtrat wesentliche oxydative Wirkungen vermissen liessen (selbst die Katalyse vou $\mathrm{H}_{2} \mathrm{O}_{2}$ blieb aus), so muss angenommen werden, dass die Substanz, mit der wir uns eben beschäftigt haben, das einzige wasserlösliche oxydirende Agens der Leberzelle, den wirksamen Bestandtheil ihres wässrigen Auszuges, darstellt.

Nach der Art der Herstellung desselben war die Annahme naheliegend, dass es mit dem von Lilienfeld ${ }^{1}$ ) aus der Thymus-

1 Zeitschrift zur physiol. Chemie Bd. 17 a. 18. 
Die Bedeut. gewisser Nucleoproteìde für d. oxydat. Leistung der Zelle. 637 drtise, von $\mathrm{H}$ ammarsten ${ }^{1}$ ) ans dem Pankreas, von Ha lliburt o ${ }^{2}$ ) aus Leber, Niere, Knochenmark and dem Stroma der rothen Blutkörperchen, von Lönnbe r g ${ }^{3}$ ) aus den Nieren hergestellten Nucleoproteïden, dem Cytoglobin von Alex. Schmidt ${ }^{4}$ ), dem Gewebsfibrinogen von Wooldridge verwandt beziehentlich identisch sein könnte. Allen diesen Präparaten war dies eine eigen, dass sie aus wässrigen Ausziigen der betreffenden Organe resp. deren Alkoholfällung (Cytoglobin) durch Zusatz sehr verdünnter Säuren als Fällungen hergestellt waren. Wir untersuchten deshalb unser Präparat genauer. Es zeigte folgende Eigenschaften:

Frisch ausgefällt und auf dem Filter gesammelt, bildete es eine graue klebrige Masse, die sich selbst überlassen eine hornartige Consistenz beim Eintrocknen annahm, nach Behandlung mit Alkohol und Aether jedoch in Form eines sehr feinen gelbbraunen Pulvers erhalten wurde. In frischem Zustande erwies sich unser Präparat in kaltem wie in heissem Wasser sehwer löslich, doch quoll es in letzterem etwas auf; es war leicht löslich in verdünnten Alkalien; die Lösungen batten in stärkerer Concentration eine hellgelbe Färbung. Aus alkalischer Lösung durch Zusatz von Säuren wieder ausgefällt, Latte die Fällung die gleicben Eigenschaften wie vorher in Bezug auf die Katalyse von $\mathrm{H}_{2} \mathrm{O}_{2}$ und die Bildung unserer synthetischen Farbstoffe. Sie war im Ueberschuss der Säure wieder löslich. Aus neutraler Lösung trat Fällung ein durch Silbernitrat, Quecksilberchlorid, Magnesiumsulfat in Krystallen. Auch diese Niederschlïge wirkten stark auf $\mathrm{H}_{2} \mathrm{O}_{2}$ und blïuten - anch olne dieses - direct die Guajactinetur. Frisch gefällt ist das Präparat löslich in sehr verdünnten ClNa- und Magnesiumsulfatlösungen.

In schwacher Sodalösung gelöst and mit $\mathrm{HCl}$ bis zu $1 \mathrm{p} . \mathrm{m}$. an Gehalt und dem gut verdauenden Glycerinauszuge der Schleimhaut eines Schweinemagens versetzt, ergab das Präparat nach 24 Stunden einen voluminösen Niederschlag, der im Laufe weiterer Beobachtung keine Abnahme mehi erfährt. Das Filtrat giebt intensive Biuretreaction, mit $\mathrm{HCl}$ und Phosphorwolframsäure Fäl-

1) Zeitschrift für physiolog. Chemie. Bd. 19.

2) The Journal of Anat. and Physiology. Vol. 13. 15. 16. 17. 18.

3) Skandinav. Árchiv f. Phys. Bd. 3. 1892.

4) Zur Blutlehre.

E. Pflnger Arehiv f. Physiologie, Bd. 67. 
lung, nicht mit Essigsäure und Ferrocyankalium. Der Niederschlag in verdünnten Alkalien löslich und durch vedünnte Säuren ausfällbar, zeigt ebenso wie das nrsprüngliche Präparat reichen Gehalt an Phosphor.

Diese Kriterien, der Gehalt an P, das Verhalten zur verdauenden Kraft des Pepsins, zu Säuren und Alkalien erscheinen völlig ausreichend, um unser Präparat als ein Nucleoproteĩd - so wollen wir nach Hammarsten's Vorgange derartige Verbindungen von Nuelein und Eiweisskörpern nennen - zu bezeichnen.

Zum Zwecke der Elementaranalyse wurde ein Präparat aus der Leber des Pferdes benutzt, das nach 3 maliger Lösung und Wiederausfällung mit absolntem Alkohol am Ruickflusskühler (zur Entfernung des Fettes ete.) gekocht, nachber mit Alkohol und Aether behandelt und schliesslich bei $110^{\circ} \mathrm{C}$. zur Gewichtsconstanz gebracht worden war.

Dasselbe ergab: $\mathrm{C}=49,12 \%$

$$
\begin{aligned}
& \mathrm{H}=7,14, \\
& \mathrm{~N}=15,55, \\
& \mathrm{P}=0,96,
\end{aligned}
$$

Es enthielt Blei schwärzenden Schwefel.

Die Asche enthielt Ca, Phosphorsäure und auffälligerweise auch Eisen. Bei näherer Prüfung zeigte es sich, dass letzteres nur nach Veraschung nachweisbar war, somit in sehr fester organischer Bindung enthaiten sein musste.

Unser Nucleoproteïd selbst gab in starker $\mathrm{HCl}$ gelöst weder mit Rhodankalium, noch mit Ferro- oder Ferridcyankalium irgendwelche Reaction, desgleichen nicht durch Kochen mit $\mathrm{KOH}$ aufgescblossen. Selbst nach 24 stündigem Steben in ammoniakalischer Lösung mitSchwefelammonium behandelt, erfolgte keine Schwarzfärbung. Auch an das Bung e'sche Reagens (10 Vol. HCl von 25\%, $90 \mathrm{Vol}$. Alkohol von $90 \%$ ) wurde Fe nicht abgegeben, selbst nach mehrstündiger Behandlung bei siedender Temperatur. Daraus geht hervor, dass das in unserm Nucleoproteïd enthaltene $\mathrm{Fe}$ weder als anorganisches Salz noch als salzartige Verbindung mit Eiweiss oder organischen Süuren $u$. dgl. enthalten war. Diesen wird durch $\mathrm{HCl}$-Alkohol unbedingt ibr Eisen entzogen. Es musste sich um eine sehr feste organische Bindung handeln, die der im Haematogen (Bunge $)^{1}$ ) entsprach.

1) Zeitschrift f. physiol. Chemie BA. 9. 49. 1885. 
Die Bedeut. gewisser Nucleoproteiđe für d. oxydat. Lejstung der Zelle. 639

Der Gehalt an Eisen betrug 0,233\% (auf die trockene Substanz bezogen).

Bestimmt warde dasselbe in der Weise, dass die Asche zunächst mit concentrirter $\mathrm{HCl}$ extrahirt, die $\mathrm{HCl}$ unter Zusatz von $\mathrm{H}_{2} \mathrm{SO}_{4}$ verdunstet, der Rückstand in Schwefelsäure gelöst und das gebildete Fe-Oxyd reducirt wurde. Dies geschah entweder durch Zufügung einiger kleinen Stückchen amalgamirten eisenfreien Zinks in einer bedeckten Ptatinschale oder nach entsprechender Verdünung in einem mit amalgamirten Zinkstücken und einem Platinblech beladenen Cylinder, wobei die verwendete Flüssigkeitsmenge die Zinkstiucke nur eben ibberragte. Titrirt wurde mit einer Permanganatlösung, deren Titre $1 \mathrm{ccm}=0,000903 \mathrm{gr}$ Fe betrug und des öfteren controlirt wurde.

1,937 des trockenen Präparates verbrauchten $5 \mathrm{ccm}$ der Permanganatlösung, somit $1,937 \mathrm{gr}=4,515 \mathrm{mgr} \mathrm{Fe}$

$$
1 \mathrm{gr}=0,233 \% \text {. }
$$

Die sonst noch untersuchten Präparate ans der Leber des Kalbes, des Rinds, des Hundes gaben ebenfalls starke qualitative Fe-Reaction (HCl und Ferrocyankalium, Rhodankalium) in der Asche.

Durch die oben angegebenen Kriterien erschien es als ausreichend sicher bewiesen, dass an der oxydativen Function der Leberzelle eine dem Zellkern angehörige, Substanz betheiligt ist, die im übrigen im wässrigen Auszuge der Organe sogar das ausschliessliche $O$ übertragende Princip darstellt. Es erscheint infolgedessen nicht mehr angebracht von einem unbekannten "Oxydationsferment" in den Geweben zu sprechen. Unsere Versuche zeigen, dass die oxydirende Kraft des wässrigen Leberextractes einer bekannten und woblcharakterisirten Verbindung, einem Nucleoproteïd, zukommt.

Sollte die Thatsache, dass dem Zellkern angehörige Substanzen eine so eigenartige Leistung entwickeln, durch weitere Beweise gestïtzt werden und der Nachweis 0 -ibertragender Kraft in unserer Verbindung nicht ein Unicum bleihen, dann müsste zunächst (für die Leberzelle) noch in erster Reibe der Nachweis geführt werden, dass auch in dem durch Wasserextraction erschöpften Zellreste die oxydative Leistung an dem Zellkern zugehörige - etwa der unserigen ähnliche Substanzen - geknüpft war.

Obgleich mit Rücksicht auf die Ausdehnung der uns gestellten Aufgabe ein eingehenderes Studium des wasserunlöslichen oxy- 
direnden Princips im Leberzellenreste nicht gut angängig war, so konnten wir zunächst feststellen, dass durch wiederholte Extraction mit sehr verdïnnten Soda- oder Ammoniaklösungen sich ans dem mit Wasser ersehöpften Zellreste eine stark $\mathrm{H}_{2} \mathrm{O}_{2}$ zerlegende Flüssigkeit gewinnen liess.

Die aus den alkalischen Extracten durch Zusatz verdünnter Mineralsäuren eintretende Faillung zeigte starke Katalyse des $\mathrm{H}_{2} \mathrm{O}_{2}$, in ausgefälltem Zustande sowohl wie in schwachem Alkali gelöst, ferner Indophenol und Toluylenblausynthese, beziehentlich Beschleunigung derselben. Sie ergab bei der Verdauung mit HCl-Pepsin einen unlöslichen Rückstand, dessen Gehalt an Phosphor 0,66\% betrug. Auch diese - in ibren sonstigen Eigenschaften - Verhalten zu Säuren und Alkalien, Fällung durch Alkohol, Eiweissreactionen etc. - der beschriebenen analoge Verbindung enthielt Eisen in organischer Bindung, nur in der Asche nachweisbar.

Eine Bestätigung geben unsere Beobachtungen $a b$ für die Untersuchungen von Alexander Schmidt1), der nach vollständiger Entfernung der in Wasser und Alkohol löslichen Bestandtheile der Leberzelle durch Behandlung der letzteren mit Alkohol eine von ihm Cytin genannte Verbindung isolirte, die der Beschreibung nach ebenfalls als Nucleoproteïd aufzufassen ist. Sie giebt einen Gehalt an $\mathrm{P}$ von $0,75 \%$ und $\mathrm{Fe} 0,19 \%$. Aus diesem $\mathrm{H}_{2} \mathrm{O}_{2}$ stark katalysirenden, in Wasser unlöslichen Nuclenproteïd spaltete A. S c h m id t durch Behandlung mit Sodalösung eine Verbindung $a b$, die mit seinem aus dem Wasserextract der Leberzelle gewonnenen Cytoglobin identisch ist. Dem letzteren gleich gab auch das aus dem Cytin gewonnene Präparat nach Säurezusatz eine $\mathrm{H}_{2} \mathrm{O}_{2}$ katalysirende Fällung (Präglobulin von $\mathrm{Sehmidt}$ ), die wohl mit unserem Nucleoproteïd verwandt ist.

Ein weiteres genaueres Studinm dieser Verbindungen, die dem in Wasser unlöslichen Antheil der Zelle angehören, soll uns demnächst ausführlich beschäftigen.

Vor der Hand hielten wir es für wesentlicher, unsere Beobachtungen über den 0 erregenden Bestandtheil des Wasserextractes der Zelle durch Untersuchung weiterer Organe zu vervollständigen, um unseren Anschauungen eine breitere Basis geben zu können.

1) a. a. 0 . 
Die Bedeut. gewisser Nucleoproteïde für d. oxydat. Leistung der Zelle. 641

\section{Das Nucleoproteïd des Pancreas.}

In der bereits beschriebenen iiblichen Weise wurde das zerkleinerte Organ des Rindes mit Wasser extrahirt und mit Säure gefällt. Durch wiederbolte Lösung in verdiunnter Sodalösung und nachherige Fällung gereinigt, ergab das erhaltene Präparat Zerlegung von $\mathrm{H}_{2} \mathrm{O}_{2}$ in einer Intensität, wie sie etwa dem ursprünglichen Gewebe zu eigen war; die Katalyse war wesentlich weniger intensiv, wie wir es an der Leber bezw. deren Nucleoproteïd gesehen hatten.

$$
\text { Versuch. }
$$

$0,2 \mathrm{gr}$ der frischen Substanz in 50 Wasser und $3 \mathrm{ccm} 1 / 10$ Normal-Sodalösung gelöst mit $10 \mathrm{ccm} \mathrm{H}_{2} \mathrm{O}_{2}$ vermischt.

$\begin{array}{lccccccccc}\text { Nach Minuten } & 1 & 2 & 3 & 4 & 6 & 7 & 10 & 18 & 25 \\ \text { ccm } \mathrm{O}_{2} \text { entwickelt } & 2 & 3,5 & 5 & 6,5 & 10 & 13 & 14 & 18 & 22\end{array}$

In gleicher Weise bildete das frisch bereitete, auf Filtrirpapier ausgestrichene Präparat Toluylenblau aus seiner. Vorstufe.

Dagegen gelang es mir nicht, erhebliche Bildung der Salicylsäure aus dem Aldehyd bei der ïbiichen Versuchsanordnung nachzuweisen. Wir erhielten in zwei Versuehen mit dem in Alkali gelösten Nucleoproteïd, das aus dem 1 Liter betragenden Wasserextracte von $200 \mathrm{gr}$ des Organs vom Rinde hergestellt war, nur Spuren von Salicylsäure $(2-3 \mathrm{mgr})$. Dies erscheint uns nicht wunderbar, wenn wir bedenken, dass auch das Pancreas selbst in toto, wie aus den Versuchen ron $\mathrm{Ab}$ el o u s und Biarnè s ${ }^{1}$, sowie von $\mathrm{Salkowski}{ }^{2}$ ) zu ersehen, diese Oxydation nur in sehr geringem Umfange zu leisten vermag. Die ersteren finden gar keine, $\mathrm{Salkowsk}$ in 2 Versuchen, in denen das Gesammtorgan verwendet worden war, je $5 \mathrm{mgr}$ Salicylsäure, während bei Verwendung des filtrirten Kochsalzauszuges nur Spuren (1 mgr) gefunden wurden.

Aus diesen Thatsachen ist zu crschliessen, dass die oxydative Kraft des Panereas, wenigstens soweit sein Wasserauszug in Frage $\mathrm{kam}$, viel geringer ist als die bis jetzt von uns an der Leber studirte, was im Uebrigen ja auch aus der schwächeren Einwirkung

1) Comptes rendus de la Soc. de Biol. 1895.

2) a. a. 0 . 
auf $\mathrm{H}_{2} \mathrm{O}_{2}$ (vgl. obigen Versuch) und dem langsameren Eintritt der Toluylenblau- oder Indophenolbildung - wie wir beobachteten und Abelous und Biarnès ebenfalls fanden - hervorgelst. Nach den Versuchen Salkow ski's musste man annehmen, dass das in Wasser unlösliche oxydirende Agens im Pancreas auch das relativ am stärksten wirksame Princip in diesem Organ repräsentire. Da es sich jedoch auch hierbei nur um Verbindungen von geringer oxydativer Energie handeln konnte, haben wir auf ein näheres Studium derselben verzichtet. Dass es sich ebenfalls um Nucleoproteïde handelte, zeigten uns analoge Versuche mit Extraction vermittels schwacher Sodalösungen, wie sie von der Leber mitgetheilt wurden.

Das aus dem Wasserextract des Panereas gewonnene Präparat glich in seinem Verhalten dem aus der Leber hergestellten, nur war es im Ueberschuss der Säure unlöslich. Es entsprach dem von $\mathrm{Hammarsten}{ }^{1}$ ) beschriebenen. Es hatte einen N.Gehalt von $17,1 \%$, einen P-Gehalt von 3,9\% (Ha mmarsten findet $17,39 \mathrm{~N}, 4,48 \mathrm{P})$. An organisch gebundenem Eisen fanden sich $0,23 \%$, also zufällig genau so viel wie im Lebernucleoproteïd. Auch Hammarsten findet seine Verbindung relativ reich an Eisen, ohne jedoch quantitave Angaben zu machen.

\section{Das Nucleoproteïd der Nieren.}

Aus den Nieren des Schweines wurde nach gründlicher Zerkleinerung ein Wasserextract gewonnen, der mit $\mathrm{HCl}$ bezw. $\mathrm{H}_{2} \mathrm{SO}_{4}$ in $1 / 10$ normaler Lösung versetzt, eine feinflockige Fällung ergab, welche nach wiederholter Reinigung $\mathrm{H}_{2} \mathrm{O}_{2}$ gut zerlegte und in der uiblichen Weise geprift, die Bildung des Toluylenblaus aus seiner Vorstufe erheblich beschleunigte. Das Präparat, das in seinem Verhalten, zu Säuren und Alkalien, zur verdanenden Kraft des Magensaftes, Eiweissreactionen den vorher aus Pancreas und Leber beschriebenen glich - es war wie ersteres auch im Säureüberschuss unlöslich - enthielt $15,5 \% \mathrm{~N}$ and $0,3 \% \mathrm{P}$.

Sein Gebalt an organisch gebundenem Eisen - und nur solches enthielt es - betrug $0,19 \%$.

Auch bei ihm war die Einwirknng auf Salicylaldehyd nicht sebr ausgesprochen, doch deutlich nachweisbar, besser als beim

1) Zeitschrift f. pbys. Chemie Bd. 19. 1884. 
Die Bedent. gewisser Nucleoproteïde für d. oxydat. Jeistung der Zelle. 643

Pancreas. Ein Versuch, in welchem der 1 Liter betragende wässrige Auszug aus $300 \mathrm{gr}$ Schweinsnieren das Nucleoproteïd geliefert hatte, ergab bei 24 stündiger Dauer aus $2 \mathrm{ccm}$ Salicylaldehyd allerdings nur 4, nach 78 Stunden jedoch $9 \mathrm{mgr}$ Salicylsäure.

Diese niedrigen Werthe stimmen ungefähr mit den Angaben Salkowski's, der Kalbsnieren verwendete, überein. Der nach Wasserextraction an dieses Lösungsmittel nur Spuren eines durch Säure fällbaren Körpers abgebende Zellrest zeigte noch gute Einwirkung auf $\mathrm{H}_{2} \mathrm{O}_{2}$ und deutliche Beschleunigung der Toluylenblausynthese. Ihm liess sich durch Behandlung mit verdiunnter Sodalösung eine Substanz entziehen, die weiter nicht eingehender untersucht wurde, jedoch in Lösung sowohl wie mit Säuren gefällt dieselben Reactionen lieferte. Auch sie wies einen Gehalt an organisch gebundenem Eisen auf.

Wenn wir aus den Versuchen Salkowski's mit Kalbsnieren, welche erweisen, dass der filtrirte ClNa-Auszug lange nicht so stark oxydirend wirkt, wie das ursprüngliche Organ selbst, einen Schluss ziehen wollen, so müssten wir annehmen, dass das im Zellreste verbleibende, in Wasser unlösliche Nucleoproteïd der Niere stärker wirksam sei als das in Wasser lösliche. Angaben über einen dem unseren analogen bezw. gleichen Körper liegen von Halliburton ${ }^{1}$ ) und Lönnberg ${ }^{2}$ ) vor. Der erstere fällte mit Essigsäure aus wässriger, letztere beiden aus ammoniak- resp. sodahaltiger Lösung. Mit dem Halliburton's stimmt auch unser aus wässrigem Auszuge erhaltenes Prïparat im P-Gehalte iiberein (Hallib. $0,36-0,38 \%$ ), während das von Lö nnberg hergestellte Präparat, anscheinend ein Gemenge eines in Wasser und eines in Ammoniak löslichen (in ersterem unlöslichen) Körpers einen höheren P-Gehalt $(0,7 \%)$ aufwies. Eisen enthielten die Präparate beider. Quantitative Angaben wurden nicht gemacht.

\section{Das Nucleoproteid des Hodens.}

Das aus den Hoden des Stieres hergestellte Präparat ergab, wenn auch in geringerem Grade als das der Nieren, deutliche Zerlegung von $\mathrm{H}_{2} \mathrm{O}_{2}$ in ausgefälltem Zustande sowohl wie nach

1) a. a. 0 .

2) a. a. 0 . 
der Lösung in Soda und deutliche Beschleunigung der Farbstoffbildung aus der Vorstufe des Toluylenblau. Ein (allerdings einziger) Versuch über die Einwirkung anf Salicylaldehyd ergab ein negatives Resultat.

Auch dieses Präparat enthielt in organisch gebundener Form $0,215 \%$ Eisen.

In Bezug auf sein Verhalten zu Säuren und Alkalien, zu Eiweissreactionen, Fällungsmitteln etc. ist Besonderes von ihm nitht mitzutheilen.

\section{Das Nucleoprotejd (Nncleohiston) der Thymus}

ist von Lilienfeld zuerst des Ausführlichen beschrieben worden, so dass eine nähere Schilderung der Eigenschaften des von uns hergestellten Präparates unterbleiben kann. Seine oxydative Leistung erwies sich deutlich an der, gat messbaren Zerlegung von $\mathrm{H}_{2} \mathrm{O}_{2}$ und in der iblichen Weise geprüft, an der Beschleunigung der Bildung des Toluylenblau. Von ihm gilt, wie für die Nucleoproteïde aller übrigen von uns bis jetzt erwähnten Organe, dass es im wässrigen Organauszuge das einzige 0 -übertragende Agens darstellte. Das Filtrat, das nach erfolgter Ausfällung des Wasserauszuges erhalten wurde, war absolut nicht im Stande, $\mathrm{H}_{2} \mathrm{O}_{2}$ zu katalysiren; höchstens entwickelten sich ein paar minimale Bläschen; die Intensität des Vorgangs war ausserordentlich geringfügig gegeniber der Zerlegung durch den urspriinglichen Wasserextract bezw. die Nucleoproteïdfällung. Auch dieses Präparat enthielt, eine Angabe, die ich bei Lilienfeld vermisse, Eisen in organisch gebundener Form. Der Gehalt betrug 0,22\% Fe.

\section{Ein Nacleoproteid ans den Zellen des Blntes.}

Nachdem für eine Reihe von Geweben nachgewiesen worden, dass die von ihnen ansgelösten oxydativen Leistnngen von Verbindungen, welche dem Zellkern zugehören, bedingt wurden, erschien es wünschenswerth nachzusehen, ob sich die ja bereits durch $\mathrm{S} a \mathrm{l}$ kowski, Abelons und Biarnès; Lẻpine etc. festgestellte gleiche Kraft des Blutes auch auf ähnliche Verbindungen zurïckführen lasse. Da es anzunehmen war, dass für die Leucocyten des Blutes das gleiche gelten konnte wie für die der Thymus und A. Schmidt schon aus den isolirten farblosen Blutkörperchen des Pferdeblutplasmas ein $\mathrm{H}_{2} \mathrm{O}_{2}$ zerlegendes Cytoglobin isolirt hatte, 
Die Bedeut. gewisser Nucleoproteide für d. oxydat. Leistung der Zelle. 645

und nm bei einer Bearbeitung des Gesammtblutes nicht Producte zu bekommen, deren Provenienz dann nicht melı zu entscheiden war, hielt ich mich allerdings zunächst an die leichter zu isolirenden rothen Blutkörperchen, konnte jedoch nicht verhüten, dass noch Reste der Lencocyten, Blutplättchen etc. denselben mit anhafteten. Es wurde etwa 1 Liter defibrinirtes Blut mit dem zelonfachen Volumen einer ClNa-Lösung versetzt, die auf 1 Volumen gesättigter (36\% ClNa-Lösung) 9 Vol. Wasser enthielt. Die Mischung liess ich in Glasschalen absetzen; nach 24 Stunden hatten sich die rothen Blutkörperchen gut abgesetzt. Dieselben wurden in derselben Weise mit der gleichen ClNa-Lösung wiederum vermischt, nach 1-2 Tagen nach Entfernung der uberstehenden Flüssigkeit mit wenig Wasser in ein Becherglas gebracht; hierauf wurde die Flüssigkeit bei $35^{\circ} \mathrm{C}$. im Wasserbade unter Zusatz von etwas Aether lackfarben gemacht. Nach Zusatz von wenigen Cubikcentimetern einer $1 / 10$ Normalsodalösung wurde die Blutflüssigkeit durch 24 Stunden sich selbst tuberlassen. Es trat nunmehr bei Zusatz einer $1 / 10$ Normalsäure eine Fällung ein, die im Ueberschuss der Säure unlöslich war.

Dieselbe wurde durch Decantiren und Filtriren auf dem Filter gesammelt, mehrfach vom Filter genommen, mit Wasser gewaschen, bis das Waschwasser keine Oxybämoglobinstreifen mehr zeigte.

Eine Spur der frischen Substanz in verdünnter Sodalösung gelöst, erwies sich dann ebenfalls frei von Blutfarbstoff. Die nach weiterem Anfösen in Soda und wiederholter Fällung gereinigte Substanz ergab, mit heissem Alcohol im Rückflusskïhler gekncht, dann mit Alcobol und Aether behandelt, ein gelbbraunes feines Pulver. In frischem Zustande geprift, zerlegte es $\mathrm{H}_{2} \mathrm{O}_{2}$ gut und ergab messbare Mengen Sauerstoffs und beschleunigte, auf Filterpapier ausgestrichen, deutlich die Synthese des Toluylenblaus.

In verdünnter Sodalösung gelöst, auf $1 / 2$ Liter aufgefüllt und mit 1 cem Salicylaldehyd versetzt, ergab sich nach 48 Stunden eine Ausbeute von $9 \mathrm{mgr}$ Salicylsäure. Es enthielt organisch gebunden $0,3 \% \mathrm{Fe}$.

Nach den Bedingungen der Herstellung des Präparates halte ich dafür, dass dieses Eisen ausschliesslich aus dem Präparate stammte und nicht aus etwaigen Verunreinigungen mit Hämoglobin oder dessen Derivaten herrührte. Die Säuremengen, die zur Ausfällung verwendet worden waren, waren so gering, dass 
irgendwelche Hämatinbildung nicht hatte eintreten können. Das Filtrat ergab nur die Streifen des Oxybämaglobins. Auch konnte ich weder aus der frisch gefällten noch mit Alcohol behandelten Substanz Häminkrystalle herstellen.

Wenn ich auch allerdings die Blutkörperchen lange mit verdinnter ClNa gewasehen hatte, kann ich, wie bereits vorausgeschickt, den Einwand nicht zuriekweisen, dass bei der Extraction mit schwachem Alkali vielleicht aus Resten weisser Blutkörperchen noch eine mit Säuren fällbare Substanz mit in Lösung gegangen und mein Präparat mit dieser verunreinigt war. Dieser Einwand kann furr uns indifferent sein, da es für uns nur darauf ankam zu zeigen, dass aus dem mit Oxydationskraft begabten Blute beziehentlich den Zellen desselben, ïberhaupt ein $\mathrm{O}$ - ï bertrage ndes Nucleoprote ïd - als solches ist unsere Substanz anzusehen - hergestellt werden konnte, das in Bezug auf Eiweissreactionen, Verhalten zu Säuren und Alkalien etc. den an den Organen beschriebenen glich. Ob dieses Nucleoproteid ausschliesslich den rothen oder aber zum Theil auch den weissen Blutzellen entstammte, konnte für unsern vorliegenden $Z_{\text {weck }}$ gleicbgültig sein.

Dass die nach anderen Methoden gut isolirten rothen Blutkörperchen ein solches enthalten, geht aus den Mittheilungen von Hallibarten ${ }^{1}$ ) and Alex Sehmidt ${ }^{2}$ ) hervor. Ersterer stellte eine $0,8 \%$ P-haltende Verbindung, die in ihrem Verbalten sonst der unsrigen glich, aus rothen Blutkörperchen von Pepton Blut her, letzterer sein Cytin, das dem aus Organen hergestellten analog war. Leider konnten aus äusseren Gründen zur Zeit meine Versuche in dieser Richtung nicht fortgesetzt werden. Es dürfte ein Leichtes sein, sie am isolirten und gereinigten Stroma zu wiederholen. Die Ausbeute aus dem Blute des Hundes war tibrigens gering. Sicherlich war unser Präparat jedoch nicht das einzige oxydirende Agens im Blute; denn das Filtrat nach erfolgter Säurefällung der aus den Blutkörperchen erhaltenen Flüssigkeit zerlegte im Gegensatz zu den wässrigen Extracten z. B. der Leber $\mathrm{H}_{2} \mathrm{O}_{2}$ noch stïrm!sch und wirkte gut glycolytisch.

Es könnte die Vermuthung nale liegen, dass vielleicht doch das Hämoglobin an der 0-Uebertragung im Blute eine wesentliche Rolle spielt. Diese Frage ist zur Zeit noch ofien und noch nicht völlig: geklärt. A. Schmidt, der dieselbe in Bezug auf die Katalyse des Superoxyds wenigstens studirt hat, findet trotz 4 maliger Reini- 
Die Bedeut. gewisser Nuclcoproteïde für d. oxydat. Leistung der Zelle. 647

gung durch Umkrystallisiren die Oxyhämaglobinkrystalle noch gut wirksam, meint aber, dass diese Wirkung ron schwer entfernbaren Stromabestandtheilen ausgehe. Ich selbst lalte dies auch nach einer gelegentlichen Beobachtung nicht für unwahrscheinlicb. Während es sonst nämlich sehr schwer gelingt, durch Zusatz selbst grosser Mengen von $\mathrm{H}_{2} \mathrm{O}_{2}$ das Blut zu entfärben - den Blutfarbstoff zu oxydiren - gelingt dies sehr leicht an dem Filtrat, das ich nach Säurefällung bei den obigen Blutversuchen erbielt. Dies beweist, dass Verbindungen, die $\mathrm{H}_{2} \mathrm{O}_{2}$ katalysiren und somit das Hämoglobin vor Oxydation schützen, im Filtrat resp. im Hämo. globin in viel geringerem Grade anwesend sind wie vor Ausfällung des (Stroma)-Nucleoproteïds. Eine Erklärung dieser Verhältnisse dürfte mit Rücksicht anf die Glycolyse und die jüngst von Cohnstein beschriebene Ueberführung von Chylusfett durch Blut in durch Aether nicht mebr nachweisbare Verbindungen (bei starker 0 -Zufuhr) nicht ohne Interesse sein.

\section{Ueber das oxydirende Agens der Muskeln}

besitze ich nur insoweit Erfahrungen, als ich mich davon überzeugt hatte, dass der ausgepresste Saft entbluteter Froschmuskeln $\mathrm{H}_{2} \mathrm{O}_{2}$ gut katalysirte, sowie dass diese mit dem Querschnitt in eine Lösung der Vorstufe des Toluylenblaus getaucht, rasch deutliche Beschleunigung der Farbstoffbildung aufwiesen, ebenso wie sie die Oxydation des Dimethylparaphenylendiamins herbeiführten (Reaction v. Wurster). Dass die oxydative Einwirkung auf Salicylaldehyd minimal war, geht aus den Angaben von Abelous und Salkowski herwor. Alexander Schmidt berichtet, dass er eine sehr geringe Menge seines Cytoglobins aus Froschmuskeln hergestellt habe.

V. Ueber die Betheiligung der Spaltungsproducte des

Nucleop roteids an der Sauerstof fübertragung.

Nachdem nunmehr für eine Reihe von Organen festgestellt war, dass deren oxydirende Kraft, zunächst, soweit es sich um ihren Wasserextract handelt, von einem Nucleoproteïd bestimmter Zusammensetzung ausging, so lag die Frage nahe, ob denn derselbe allein und ausschliesslich 0 übertragend wirke, oder ob etwa

1) The Journal of Anat. and Physiology Vol. 18.

2) a. a. 0. 
diese Function an einzelne bestimmte Theile dieses complicirt gebauten Gebildes, dessen Constitution und Abbau wir nunmehr durch die Untersuchungen Kossel's kennen, gebunden sei.

Wie Lilienfeld an seinem Nucleoproteïd der Thymus gezeigt hat, kawn dasselbe - sein Nucleohiston - durch eine Reihe verschiedenartiger Eingriffe, wie Behandeln mit $0,8 \% \mathrm{HCl}$, mit Barythydrat, durch Kochen mit heissem Wasser; schliesslich durch die Verdauung mit HCl-Pepsin in ein Nucleïn und ein von ihm als Histon bezeichneten Eiweisskörper gespalten werden. Da alle diesse Eingriffe bis auf den letzten wegen der grossen Empfindlichkeit der oxydativen Function der Nucleoproteïde dieselbe vollständig vernichteten und somit ihren Nachweis illusorisch machten, so blieb als relativ barmloser Eingriff nur die Verdauungsmethode uibrig.

Da sich aber selbst $1 \%$ Lösungen des HCl nicht als gleichgültig erwiesen und die zulässige Grenze, bis zu der die Katalyse des $\mathrm{H}_{2} \mathrm{O}_{2}$ noch ungeschwächt erfolgte, etwa einem Gehalt von $3 \mathrm{ccm}$ $1 / 10$ normal. $\mathrm{HCl}$ auf $50 \mathrm{cem}$ Wasser $=0,216 \%$ entsprach (bei einem Gehalt von 0,2 Substanz), so mussten Lösungen dieser Concentration verwendet werden.

$\mathrm{Zu}$ diesen Versuchen warden die Nucleoproteïde der Leber, des Pancreas und der Thymus verwendet, die alle gleichmässig das gleiche Verbalten boten und infolgedessen eine zusammenhängende Darstellung erfabren können.

Eine geringe Menge des frischen Nucleoproteïds wurde mit Hülfe von wenig Alkali in $50 \mathrm{ccm}$ Wasser gelöst und $\mathrm{HCl}$ bis zu einem Gehalt von $0,2 \%$ an freier Salzsäure und $1 \mathrm{ccm}$ eines gut verdauenden Pepsinglycerinauszuges vom Schweinemagen sowie etwas Thymol zugefügt. Nach 24 Stunden hatte sich cin starker Niederschlag ausgesehieden, der nach 48 Stunden nur eine geringe Vermehrung erfahren hatte. Er hatte, in schwacher Sodalösung gelöst, nicht mehr die Fähigkeit, aus einer $\mathrm{H}_{2} \mathrm{O}_{2}$-Lösung irgendwie wesentliche Mengen $\mathrm{O}_{2}$ zu entwickeln. Nach Verlauf von 24 Stunden waren im Eudiometerrohre nur $2 \mathrm{ccm} 0$ zu messen. Doch besass er noch insoweit einen Rest der friberen Kraft, dass er in Substanz - aus seiner Lösung mit Säure ausgefällt das Superoxyd (qualitativ durch die Guajacreaction nachweisbar) zerlegte. Diese Reaction war in Lösung nicht zu erzielen. Er zeigte also noch die "Contactwirkung". 
Die Bedeut. gewisser Nucleoproteide für d. oxydat. Leistung der Zelle. 649

Die Beschleunigung der synthetischen Bildung der Indophenole und des Toluylenblaus vermochte das den Verdaungsrückstand repräsentirende Nucleïn nicht zu liefern. Seine Kraft reichte nicht melr aus, ein $\mathrm{O}_{2}$-Molecull zu activiren; hingegen lieferte es die Farbstoffsynthesen vermittels $\mathrm{H}_{2} \mathrm{O}_{2}$.

Unser Nucleïn aus dem Leberproteid wies einen Gebalt von $1,2 \% \mathrm{P}$ auf und enthielt $0,12 \% \mathrm{Fe}$.

Die bezigglichen gleichen Producte aus Pancreas und Thymus wurden nur qualitativ auf Fe gepruft mit starkem positiven Resultat.

Das Filtrat des Nucleinniederschlages enthielt das aus dem verdauten Eiweissbestandtheil des Nucleoproteïds (H is to n, L i li e $\mathrm{n} \mathrm{feld}$ ) gebildete Pepton. Mit $\mathrm{HCl}$ und Phosphorwolframsäure oder Quecksilberchlorid ausgefällt, entbehrte dasselbe jeder Einwirkung auf die Farbstoffcomponenten und zeigte auch bei der Prïfung mit $\mathrm{H}_{2} \mathrm{O}_{2}$ keine "Contactwirkung".

Da wir nun gesehen hatten, dass die maximale oxydative Leistung, wie sie von den Nucleoproteïden der einzelnen Organe geleistet wurde, ausschliesslich an ihre Integrität gebunden war, dass das durch die Verdauung abgespaltene Nucleïn zunächst bis auf die erwähnte "Contactwirkung" jeder weiteren oxydativen Kraft ermangelte, wurde von dieser Methode der Trennung nunmehr abgesehen und den Angaben Lilienfeld's entsprechend durch Behandlung mit $\mathrm{HCl}$ von $0,8 \%$ resp. mit Barythydrat eine Spaltung des Nucleïns vom Proteïd versucht; dies konnte um so eher geschehen, als Lilie $\mathrm{n} f$ eld für sein Präparat wenigstens die Identität der auf diesen verschiedenen Wegen hergestellten Producte bewiesen hatte.

Die Behandlung mit Barythydrat lieferte ein stark P.haltiges Nuclein, das ebenfalls in ungelöstem Zustande $\mathrm{H}_{2} \mathrm{O}_{2}$ sebr schwach, doch deutlich katalysirte. Ebenso verhielt sich ein Präparat, das durch Bebandlung mit $0,8 \% \mathrm{HCl}$ gewonnen worden war.

Beide Nucleïne erweisen sicb als stark eisenhaltig.

Zur Herstellung des neben dem Nuclein bei der Spaltung des Nucleoproteïds entstehenden Eiweisskörpers bediente ich mich ebenfalls der Behandlung mit $0,8 \%$ HCl. 36 gr des Nucleoproteïds der Leber wurden zu diesem Versuche durch je 3 Stunden wiederholt auf dem Wasserbade bei $40^{\circ} \mathrm{C}$. extrahirt. Der dritte Auszug ergab keine Ausbeute mehr. Die vereinigten Extracte wurden mit 
sehr verdünntem Ammoniak gefällt, wobei sich die feinflockige weisse Fällung als im geringsten Ueberschusse desselben löslich erwies. Die gewonnene Verbindung ergab Rothfärbung mit Mill o n's Reagens in der Wärme, Biuretreaction schon in der Kälte, Fällung mit Ammoniumsulfat. Sie erwies sich frei von Phosphor und enthielt in der Asche noch deutlich nachweisbar Eisen. Diese Verbindung zerlegte $\mathrm{H}_{2} \mathrm{O}_{2}$ noch in ungelöstem Zustande deutlich, jedoch nur in diesem - selbst nach dem Erwärmen auf $80^{\circ}$-, sie bildete dagegen kein Toluylenblau mehr. Am besten katalysirte das $\mathrm{H}_{2} \mathrm{O}_{2}$ das Histon der Leber, dann das des Pancreas, am schwächsten war die Einwirkung des Präparates aus der Thymus.

Nachdem durch diese Versuche wiederholt erwiesen war, dass beide Spaltungsprodukte des ursprünglichen Nucleoproteïds noeh jenen Rest oxydativer Kraft besassen, der sich in der qualitativ noch nachweisbaren Katalyse des $\mathrm{H}_{2} \mathrm{O}_{2}$ durch die ungelöste Substanz iusserte, erschien es interessant, die weiteren Spaltungsprodukte unserer Verbindung in Bezng auf ihre katalytische Kraft zu prïfen. Nachdem das P-freie Histon sich als gleichfalls, wenn auch schwächer als das Nuclë̈n, wirksam erwiesen hatte, musste die Annahme von der Hand gewiesen werden, dass die oxydative Kraft etwa dem P-haltigen Bestandtheil unserer Verbindung immanent wäre und etwa auf eine eigenthümliche Bindung oder Lagerung des Phosphors bezogen werden könne. Vielmehr erschien der wiederholte constante, nunmehr auch im Histon erhobene Befund von organisch gebundenem Eisen interessant genug, um in Erinnerung an die $\mathrm{O}$ übertragende Kraft gewisser anorganischer Eisensalze (Katalyse von $\mathrm{H}_{2} \mathrm{O}_{2}$ durch Eisensulfat etc.) weitere Spaltungsprodukte unserer Verbindung auf ihren Gehalt an Eisen sowobl, wie auf ibre katalaytische Kraft zu untersuchen.

Ich betone an dieser Stelle wiederholt, dass wir den von uns der Einfachheit halber als Contactwirkung bezeichneten Rest oxydativer Kraft, der den Spaltungsprodukten verblieben war, selbstverständlich nicht bloss auf einen von der feinen Vertheilung abhängigen Contact zurïckfuihren, sondern dass in erster Reihe eine spezifische, besondere Eigenschaft derselben angenommen werden muss, die allen anderen Eiweisskörpern etc. abgeht.

Um zunächst die Nucleïnsäure zn prufen, ging ich vom Nuclë̈n der Pferdeleber aus, welches aus dem Nucleoproteïd durch Behandeln mit $0,8 \% \mathrm{HCl}$ gewonnen worden war. Den Angaben 
Die Bedeut. gewisser Nucleoproteide für d. oxydat. Leistung der Zelle. 651

K o s s e l's bezüglich der Herstellung der Nucleïnsäure aus Thymus entsprechend, behandelte ich $30 \mathrm{gr}$ des Nucleïns mit Barythydrat in dem von Kos s 1 angegebenen Verhältniss, und fällte das Eiweiss mit Essigsäure aus. Die auf dem Paraffinbade mit Wasser ausgekochte Barytfüllung wurde mit sebr verdiunntem HCl-Alcohol gefällt; sie war im Ueberschuss löslich. Es schied sich ein feines weisses Pulver aus, das mit Alcohol und Aether gewaschen, $\mathrm{H}_{2} \mathrm{O}_{2}$ wenn auch schwach zerlegte und in der Asche Fe-Reaction ergab.

Um das aus dem Nuclein neben der Nucleinsäure entstehende Eiweiss zu untersucben, wurde eine grössere Menge des Nucleïns mit concentrirter $\mathrm{KOH}$ zerlegt und nach einiger Zeit mit concentrirter Essigsäure übersäuert; die massige flockige Eiweissfällung ergab nach dem Lösen in Alkali mit Essigsäure und Ferrocyankalium, mit $\mathrm{HCl}$ und Glaubersalz, mit Salpetersäure und Kochen Niederschläge.

Zar Entfernung der Nucleïnsäure wurde der Niederschlag noch einmal in verdiunnter Sodalösung gelöst, mit Essigsäure gefällt und mit essigsäurehaltigem Wasser gewaschen, dann noch einmal in verdünnter $\mathrm{HCl}$ gelöst und aus dieser Lösung mit Ammonsulfat gefällt. Der so erhaltene Eiweisskörper zeigte die Charaktere eines Alkalialbuminats, es zerlegte deutlich $\mathrm{H}_{2} \mathrm{O}_{2}$ und enthielt in seiner Asche Eisen.

Zur vollständigen Aufschliessung des Nucleïns wurde dasselbe durch 18 Std. am Rückflusskühler auf dem Paraffinbade mit Wasser stark erhitzt; der bleibende Rückstand zeigte jedoch noch Zerlegung von $\mathrm{H}_{2} \mathrm{O}_{2}$. (Deutliche Fe-Reaction in del Asche.) Erst nach 2 stündigem Kochen mit $4{ }_{i}^{\circ} \mathrm{H}_{2} \mathrm{SO}_{4}$ auf dem Sandbade, wobei das Filtrat deutlich Phosphorsäure aufwies, ergab sowohl der Riuckstand, wie das eingedampfte Filtrat nicht die geringste Einwirkung auf $\mathrm{H}_{2} \mathrm{O}_{2}$ mehr $^{1}$ ).

VI. Ueber die Bedeutung des in den Nucleoproteïden organiseh gebundenen Eisens.

Nachdem wir nunmehr die wesentliche Rolle bestimmter woblcharakierisirter Zellkernsubstanzen für die oxydative Leistung

1) Die in obigen Versuchen festgestellte Thatsache, dass den einzelnen Spaltungsproducten des Nucleoproteïds immer noch Eisen zugehört, zeigt uns zugleich, welche ausserordentliche Grösse das ursprüngliche Molecül besitzen muss. 
der Organe und der sie zusammensetzenden Zellen nachgewiesen und auch hatten zeigen können, dass sogar den einzelnen Spaltungsprodukten derselben, wenn auch in sehr geringfügiger Weise (Con. tactwirkung), eine "O-erregende" Wirkung zukommt, ist die Frage des Nähern zu erörtern, wie wir uns denn jene eigenthümliche Funktion unserer Nucleoproteïde zu erklären haben. Bleiben wir auf dem Boden der von uns vertretenen $\mathrm{T} r$ a u b e'schen Anschauung, dann werden wir in allgemeiner Form sagen müssen, dass unsere Z' 'lkernsubstanzen kraft ihrer spezifischen und eigenartigen Constitution beziehentlich der Anwesenheit bestimmter Atomgruppen, befähigt sind, molecularen Sauerstofi zu activiren, ähnlich wie gewisse anorganische Substrate dies thun. Und wenn wir für jene Besonderheit nach weiteren Einzelbeiten suchen, die uns zur Zeit ja völlig unbekannt sind, so werden wir an dem constant nachgewiesenen Befund organisch gebundenen Eisens in ihnen und ihren Spaltangsprodukten wohl nicht gleichgulltig vorübergehen können. Wir werden uns des Gedankens nicht entscblagen können, dass das im Molecul in eigenartiger organischer Form wohl an Coder CN-Gruppen gebundene Eisenatom eben kraft der Eigenart seiner Bindung im Stande ist, jeweils 0 aufzunehmen und abzustossen, sich in Anwesenheit von molecularem $O$ abwechselnd zu oxydiren und zu reduciren, die frei werdenden 0 -Atome an schwerer oxydirbare Verbindungen zu übertragen, sowie wir uns ja auch die Katalyse des $\mathrm{H}_{2} \mathrm{O}_{2}$, sowie die $\mathrm{O}$-Uebertragung durch anorganische Erreger durch deren abwechselnde Oxydation und Reduction vorstellen. Unsere Vermuthung über die Rolle des Eisenatoms stützt nicht nur sein constanter Befund, sondern anch die Thatsache, dass bei der Spaltung des Moleculs, so lange sich noch grössere Atomcomplexe, die alle Eisen enthalten, erhalten haben, eine wenn auch sehr geringe O-Uebertragung noch zu Stande kommt. Erst in dem Angenblicke, wo der Zusammenhang des Moleculs vollständig gesprengt wird und also auch jene organische Bindung des Eisen-Atoms, die wir ja verantwortlich machen, aufgelöst wird, ist der letzte Rest $\mathrm{O}$-übertragender Kraft verschwunden.

Wir müssen ferner annehmen, dass die eisenhaltige organische Gruppe im Nucleoproteid eine andere Configuration besitzt als die seiner Spaltungsproducte, entsprechend der ausserordentlich verschiedenen Intensität in der sauerstoffibertragenden Wirkung beider. Die Atome miissen sich in ihr in einem sehr labilen Gleichgewicht 
Die Bedeut. gewisser Nucleoproteïde für d. oxydat. Leistung der Zelle. 653

befinden, da schon sebr schwache chemische Agentien die sanerstoffübertragende Wirkung der Nucleoproteide stark beeinflussen.

Die Differenz in der oxydativen Kraft der einzelnen Zellarten werden wir nach unseren Analysen nicht wohl auf einen wesentlich verschiedenen Gehalt an gebundenem Eisen beziehen können, sondern nur annehmen dürfen, dass nach dem relativ verschiedenen Bau der einzelnen Nucleoproteïde - wie er sich in grobem Verhältnisse ja sehon in den Differenzen ihrer elementaren Zusammensetzung zeigt - die Art der Bindung eine andere, nehr oder weniger für die O-Uebertragung geeignete ist.

So wesentlich wir die Rolle des in den spezifischen Verbindungen des Zellkerns gebundenen organischen Eisens halten, so nehmen wir andererseits selbstverständlich nicht im entferntesten an, dass jedes organisch gebundene Eisenatom in irgend welchen anderen Substraten 0 -erregend wirken müsse.

So fanden wir weder am Hämatogen des Hühnereies, resp. am Dotter desselben, das sein Eisen auch in Form einer Nucleoalbuminverbindung enthält, noch am Ferratin $S$ c h m i ed eb e r g's, dem künstlich hergestellten Analogon der aus dem Wasserextracte der Leber herstellbaren Ferrialbuminsäure, das nach Schmiedeberg auch sein Eisen in organischer Bindung trägt, die allergeringste $\mathrm{O}$-übertragende, oder auch nur $\mathrm{H}_{2} \mathrm{O}_{2}$ zerlegende Thätigkeit.

Wir meinen auch nicht etwa, dass überall $\mathrm{da}$, wo in thierischen oder pflanzlichen Stoffen Oxydationen zu Stande kommen können, unter allen Umständen $\mathrm{O}$-Uebertragung nur durch Zellkernsubstanzen resp. durch das in ihnen enthaltene Eisenatom, und nicht auf irgend einem anderen Wege, zu Stande' kommen könne.

Wir präcisiren unsere Anschauung nur dahin, dass wir die jetzt bekannten durch thierische Zellen ausserhalb des Organismus bedingten Oxydationsvorgänge auf den Gehalt jener Zellen an wirksamen Nucleoproteïden von eigener Art resp. auf eine die O-Uebertragung vermittelnde organische Bindung des Eisens in ibnen zurückfiùhren.

Mit unseren Versuchen hätte eine Theorie wesentliche und neue Stützen erhalten, die zuerst für den lebenden Organismus, wenn auch nur vermuthungsweise (ohne weitere Grundlagen) -, ausgesprochen $\mathrm{zu}$ haben, $\mathrm{B} \mathfrak{u} \mathbf{n} \mathrm{ge}^{\mathbf{1}}$ ) das Verdienst besitzt.

1) Lehrbuch $d$. physiolog. Chemie.

E. Pfinger, Arehiv für Physiologie. Bd. 67. 
VII. Ueber die reducirende Fähigkeit der $\mathrm{Nucleoproteide.}$

In unserer früheren Arbeit schon hatten wir darauf anfmerksam gemacht, dass ausser den Oxydationsvorgängen von todten Substraten bei ungenügender 0 -Zufuhr oder bei O-Abschluss Reductionsprocesse ausgelöst werden können. Wir hatten zur Erklärung derselben angenommen, dass ausser den 0 -erregenden auch reducirende Substanzen in ihnen vorkommen. Bei der Darstellung unserer Nucleoproteïde konnten wir uns davon überzeugen, dass diese selbst neben ihrer Fähigkeit, $O$ bei maximaler Luftzufuhr zu iibertragen, bei Luftabschluss auch reducirend wirkten. Ein Nucleoproteïd, das in ausgefälltem Zustande sehr rasch bei Luftzutritt die Farbstoffsynthesen lieferte, wurde mit Hülfe von wenig Soda in durch Kochen ron Luft möglichst befreitem Wasser gelöst und hierzu eine Toluylenblaulösung gebracht, das bis zum Rande fast gefuillte Reagensglas durch eine Kautschukkappe geschlossen. Nach einiger Zeit erschien der grössere untere Theil der Flüssigkeit farblos, während oben eine gefärbte Zone sich befand. Beim Schütteln mit Luft wurde die entfärbte Partie wieder gefärbt. Die Färbung verschwand bei ruhigem Stehen und Luftabschluss wieder nach einiger Zeit. Das zur Controle verwendete, $\mathrm{H}_{2} \mathrm{O}_{2}$ nicht mehr zerlegende Filtrat nach erfolgter Ausfällung des Proteïds aus dem Wasserextracte der Leber zeigte diese reducirende Wirkung nicht.

Diese Thatsache zeigt uns, dass oxydirende und reducirende Kräfte der Zelle - wenigstens soweit es sich zunächst um ibre wasserlöslichen Bestandtheile handelt - in einem und demselben Substrate vereinigt sind. Diese Beobachtung nähert uns in einem gewissen Punkte der theoretischen Anschauung Hoppe-Seyler's und wird uns vielleicht $z u$ einem weiteren Verständniss der Mechanils der postmortalen (und möglicherweise der intravitalen) Oxydationsvorgänge den Weg zeigen.

Wir batten die Annahme Hoppe-Seyler's, dass reducirende Substanz in den todten Substraten wie in der lebenden Zelle die Oxydationen bedingen, um deswillen bekämpft, weil wir ja unter reducirenden Verbindungen solehe verstehen, die den von ihnen aufgenommenen 0 in fester Bindung ihrem Molecul einverleiben und dort festhalten. Zu- 
gegeben, dass bei dieser Gelegenheit aus dem 0-Molecul frei werdende $\mathrm{O}$-Atome sich an andere dysoxydable Körper begeben, so muss ja unbedingt angenommen werden, dass die 0 -aufnehmende, reducirende Verbindung in dem Augenblicke, in dem sie $O$ aufnimmt und festhält, definitiv ihre Fähigkeit verliert, wieder zu reduciren. Es missste somit in todten Substraten nach völliger Oxydation der reducirenden Substanzen schliesslich ein Punkt eintreten, in dem die Gewebe die Fähigkeit zu oxydiren ubberbaupt nicht mebr besitzen. Und gerade die Persistenz der oxydativen Kraft, die immer neue Mengen oxydabler Substanzen von den Geweben oxydirt werden lässt, bildet ja das Charakteristische des Vorganges und findet durch die Theorie von Tra u be die durch $\mathrm{H}$ o p pe-Se y le r nicht gegebene Aufklärung.

Unsere Beobachtungen über die reducirende Kraft unserer sonst $\mathbf{0}$-übertragenden Verbindung veranlassen uns zu der Annahme, dass der Reduction bei Luftabschluss wie der O-Uebertragung bei maximalem Luftzutritt in dem gleichen Substrate die gleiche Ursache, d. h. die gleiche Atomgruppirung zu Grunde liegen dürfte. Reduction und Oxydation wird bedingt durch die in dem ersteren Falle fehlende, in dem zweiten vorhandene Massenwirkung atmosphärischen Sauerstoffs.

Inwieweit die an todten Substraten gewonnenen Erfahrungen auf die Verhältnisse im lebenden Organismus zu übertragen sind, lässt sich zur Zeit noch nicht sagen. Wir werden besonders mit Riicksicht auf die bekannten Versuche von P. Ehrlich die Wahrscheinlichkeit ganz gewiss nicht von der Hand weisen dürfen, dass auch in der lebenden Zelle den unseren analoge wohl complicirter gebaute Verbindungen die O-Aufnahme und -Uebertragung bedingen.

Es ist wohl sicher anzunehmen, dass diese Verbindungen einen wesentlich complicirteren Bau aufzuweisen haben, da wir ja durch die Extraction mit Wasser, welche für den Aufbau der Zelle gewiss keinen gleichgïltigen Eingriff darstellt, nur einen aus dem Zusammenhang gerissenen mehr oder weniger grossen Atomcomplex isoliren, der sich mit einer Reihe ähnlich gebauter, wie die Versuche am todten Substrat ja schon zeigen, in Verbindung befindet.

Unsere neue Erkenntniss ist um so interessanter, als wir ja trotz umfassender Erfahrungen über die Bedeutung des Kerns für 
Bau und Entwicklung der Zelle, über seine Function für den Stoffwechsel desselben bis jetzt keine Vorstellung hatten.

Wenn ich die Resultate meiner Untersuchungen, die in vielen Punkten noch weiterer Bearbeitung bedürftig sind, jetzt schon der Oeffentlichkeit übergebe, so geschieht dies, um auf einem so interessanten Gebiete, wie es die Biochemie der Zelle ist, zur Mitarbeit anzuregen. 

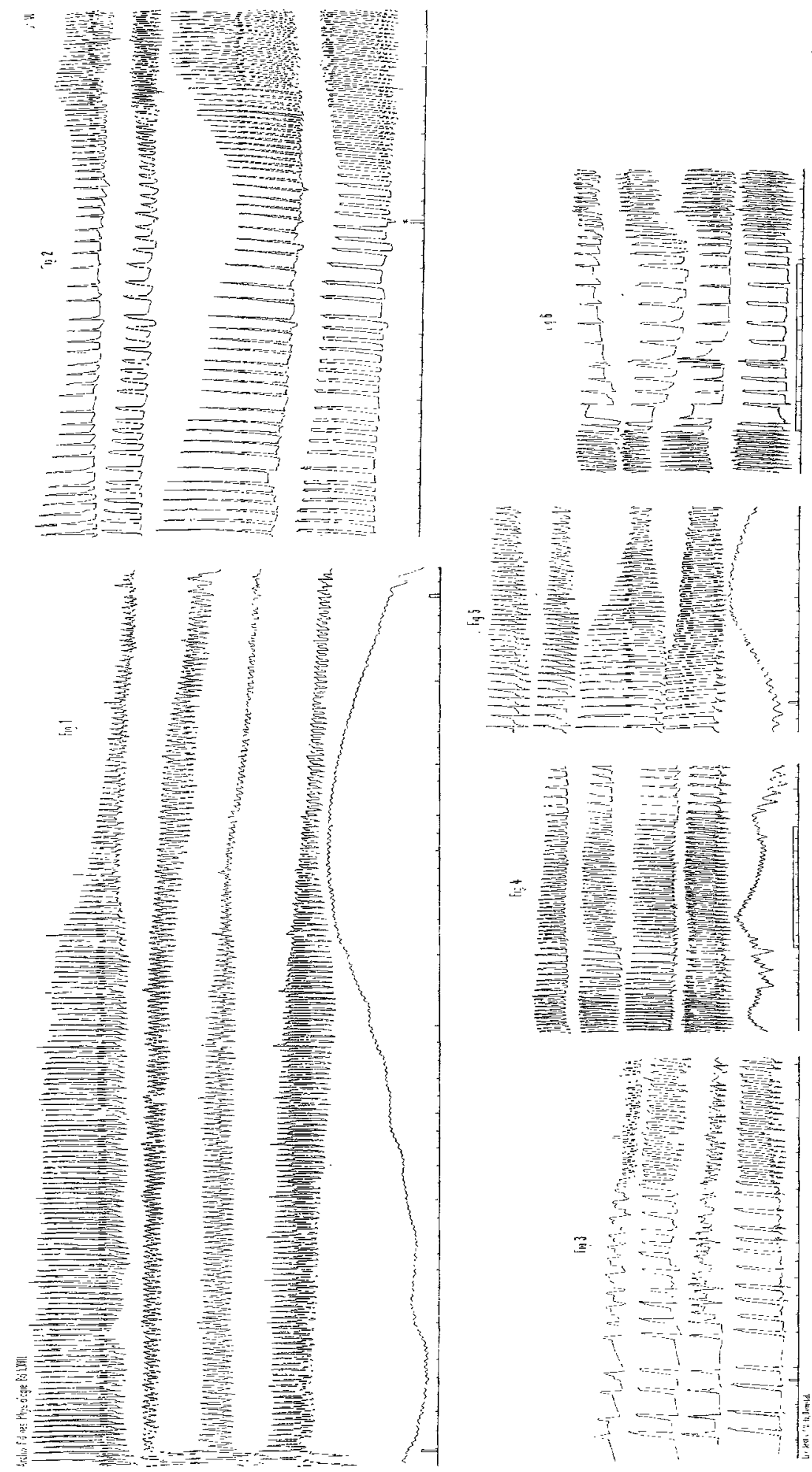

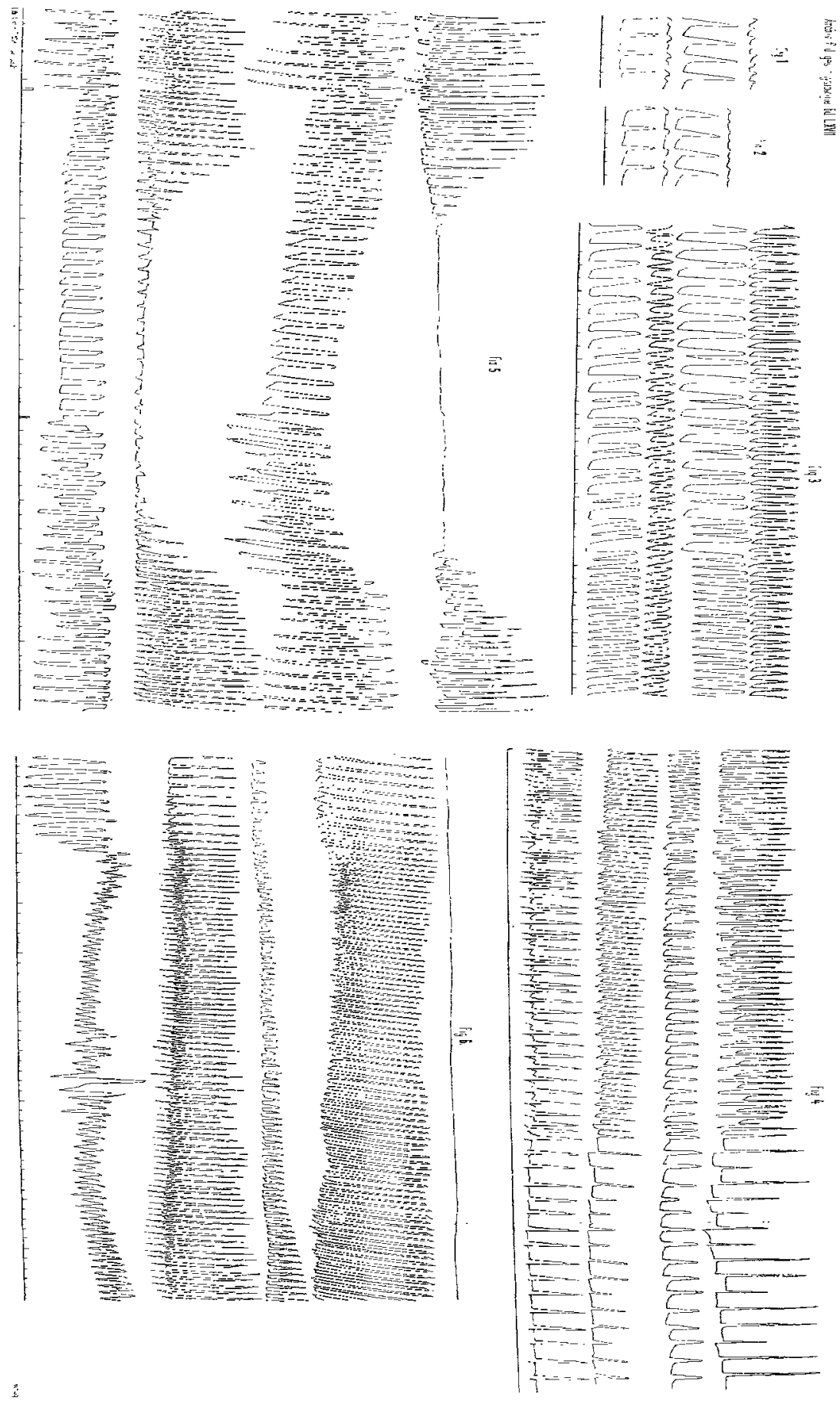


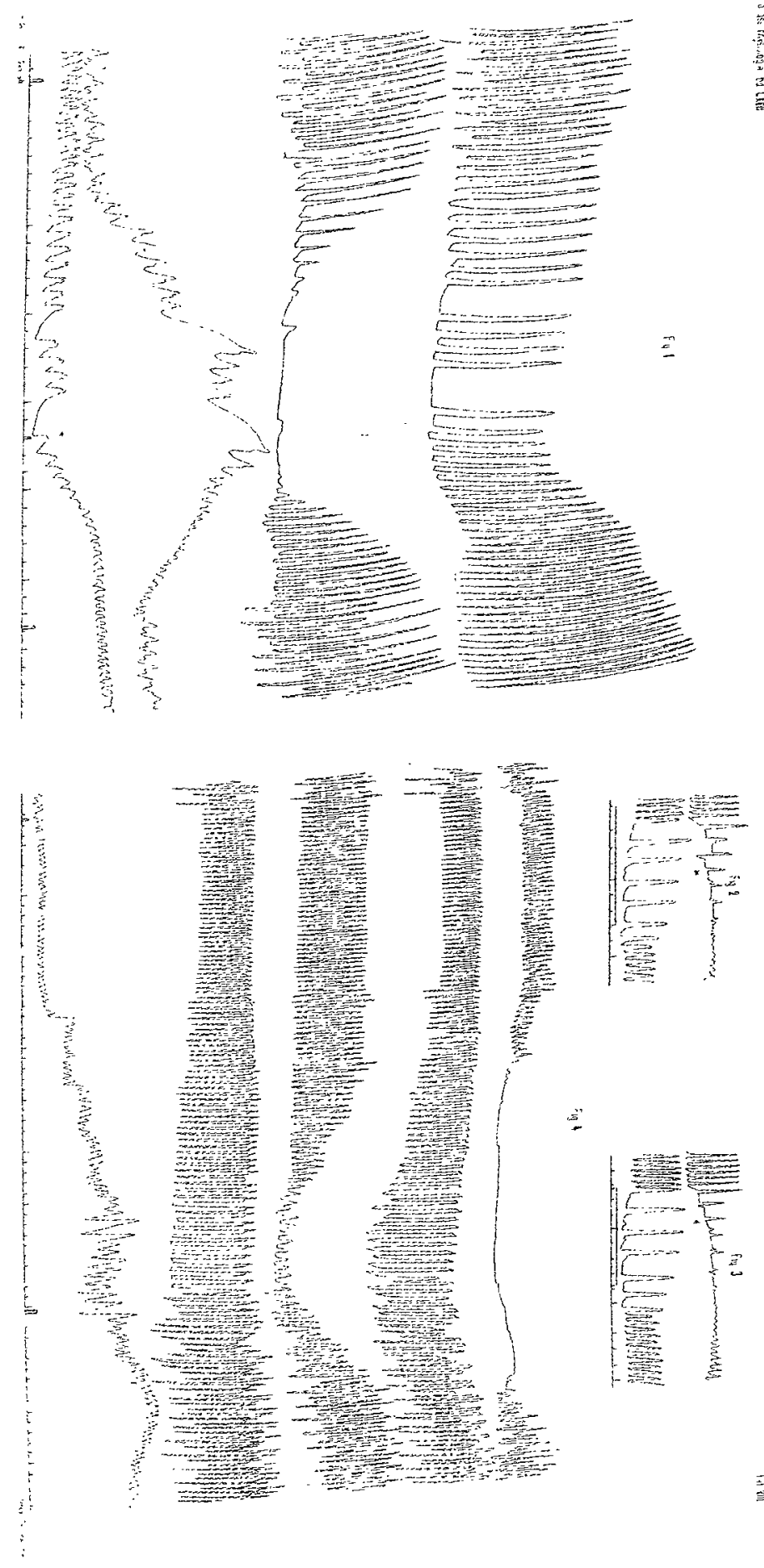



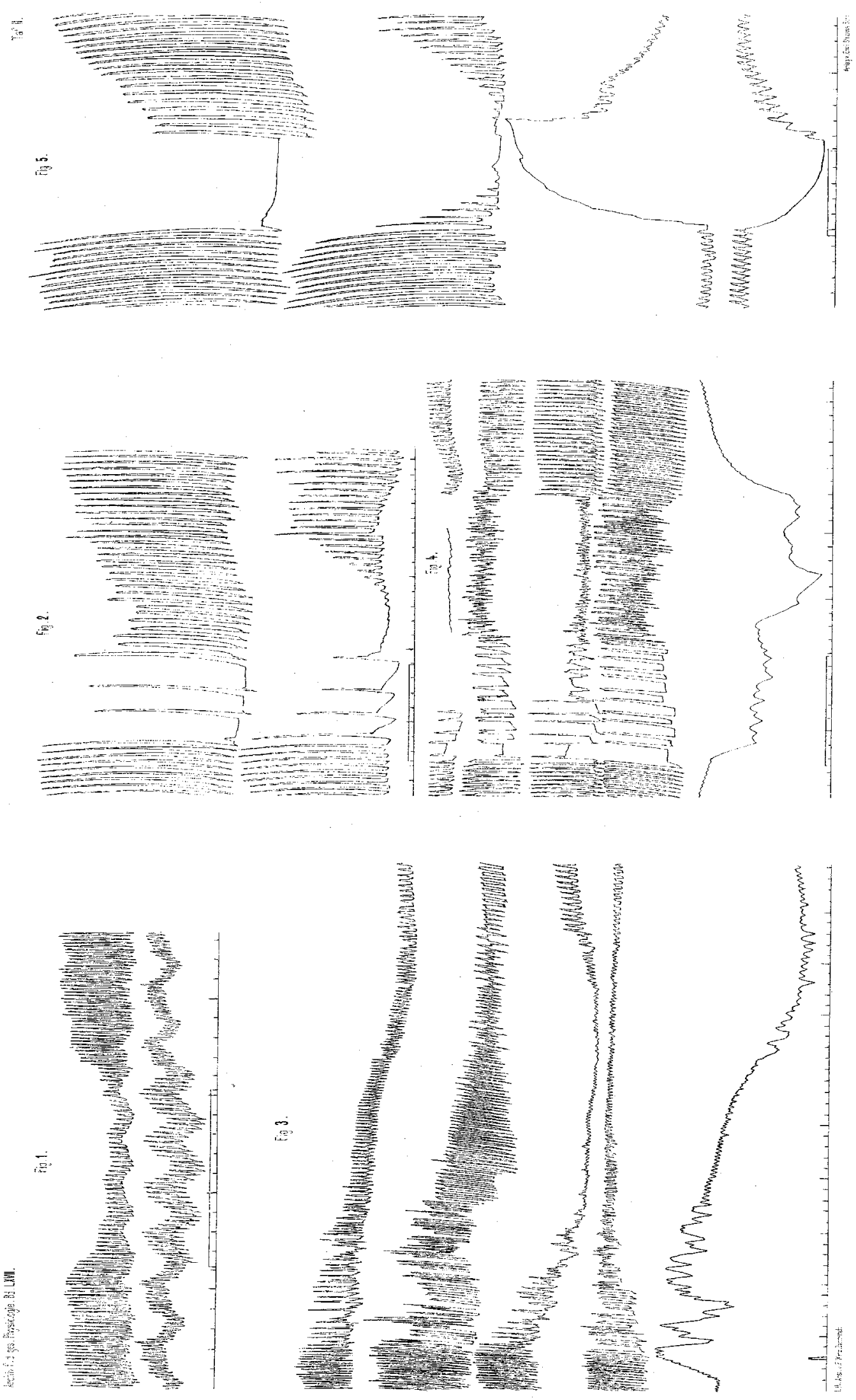


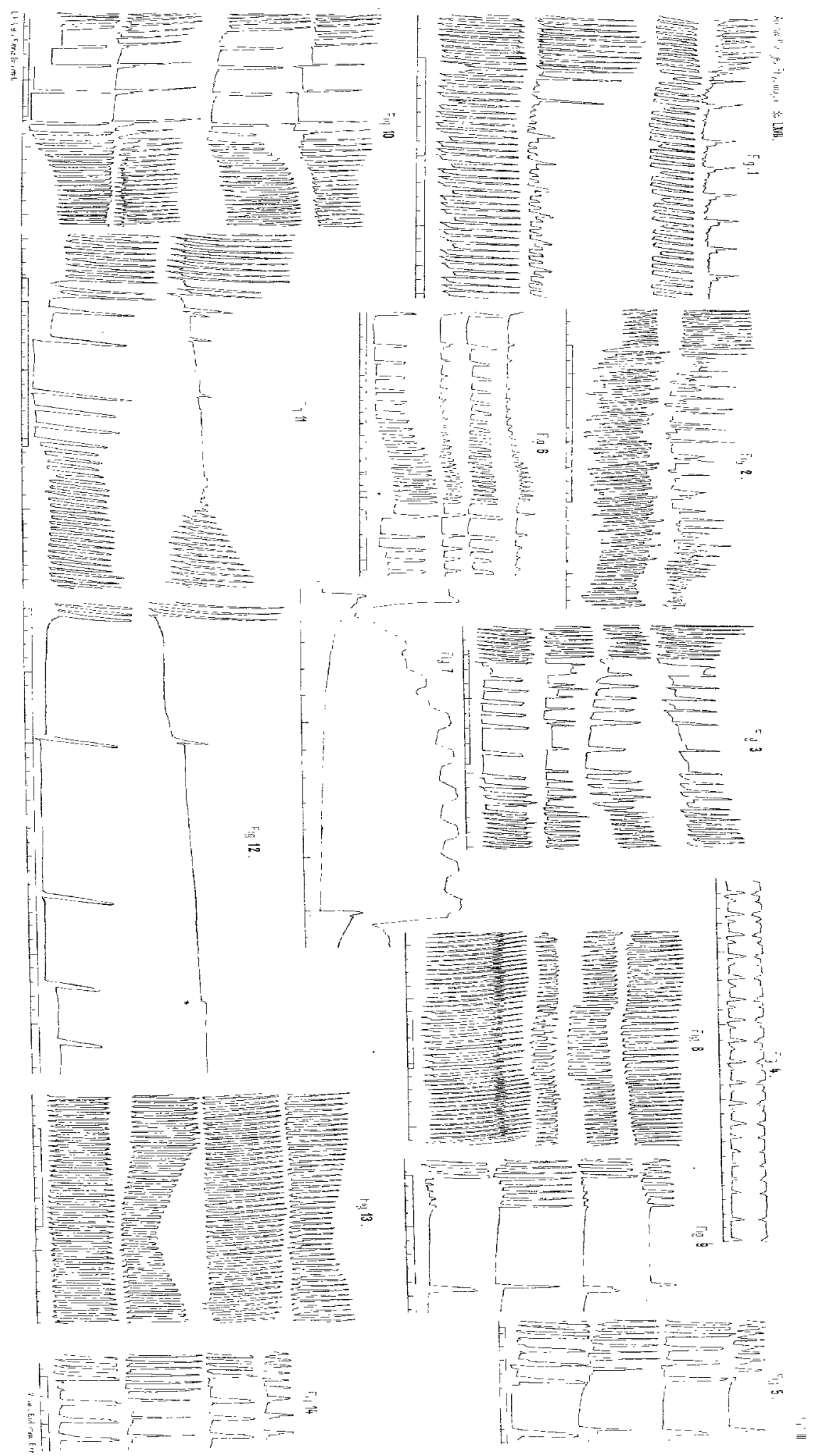




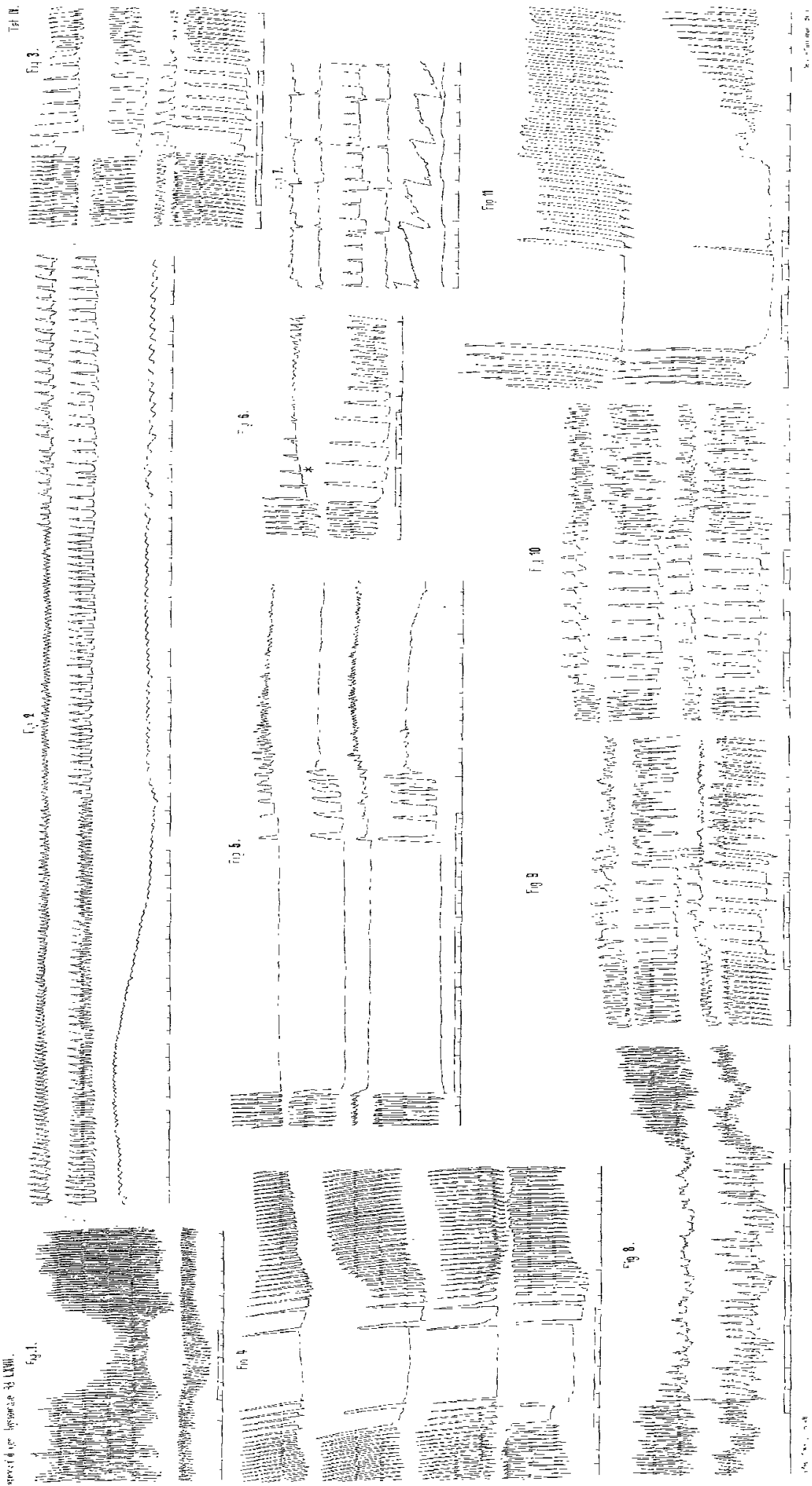




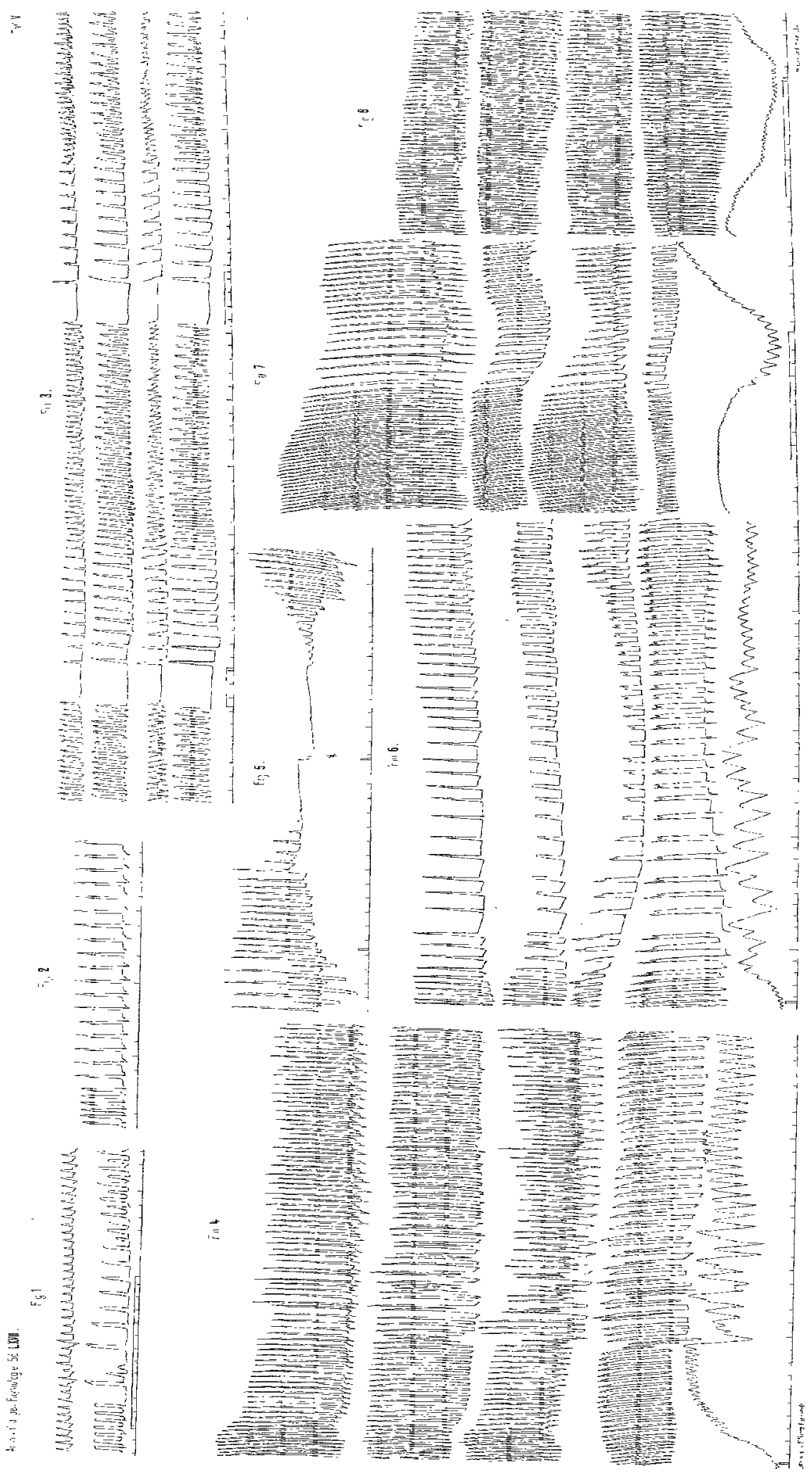

